\title{
The Fossil Nuclear Outflow in the Central 30 pc of the Galactic Center
}

\author{
Hsieh, Pei-Ying ${ }^{1,2}$, Ho, Paul T. P. ${ }^{1,3}$, Hwang, Chorng-Yuan² ${ }^{2}$, Shimajiri, Yoshito ${ }^{4,5}$, \\ Matsushita, Satoki ${ }^{1}$, Koch, Patrick M. ${ }^{1}$, \& Iono, Daisuke ${ }^{5,6}$ \\ pyhsieh@asiaa.sinica.edu.tw \\ ${ }^{1}$ Academia Sinica Institute of Astronomy and Astrophysics, P.O. Box 23-141, Taipei \\ 10617, Taiwan, R.O.C. \\ ${ }^{2}$ Institute of Astronomy, National Central University, No.300, Jhongda Rd., Jhongli City, \\ Taoyuan County 32001, Taiwan, R.O.C. \\ ${ }^{3}$ East Asian Observatory, 660 N. Aohoku Place, University Park, Hilo, Hawaii 96720, \\ U.S.A. \\ ${ }^{4}$ Laboratoire AIM, CEA/DRFCNRSUniversité Paris Diderot, IRFU/Service \\ d'Astrophysique, C.E. Saclay, Orme des Merisiers, 91191 Gif-sur-Yvette, France \\ ${ }^{5}$ National Astronomical Observatory of Japan, 2-21-1 Osawa, Mitaka, Tokyo 181-8588, \\ Japan \\ ${ }^{6}$ The Graduate University for Advanced Studies (SOKENDAI), 2-21-1 Osawa, Mitaka, \\ Tokyo 181-8588, Japan
}

Received ; accepted 


\begin{abstract}
We report a new 1 -pc $\left(30^{\prime \prime}\right)$ resolution $\operatorname{CS}(J=2-1)$ line map of the central 30 pc of the Galactic Center (GC), made with the Nobeyama 45m telescope. We revisit our previous study of the extraplanar feature called polar arc (PA), which is a molecular cloud located above SgrA* with a velocity gradient perpendicular to the Galactic plane. We find that the PA can be traced back to the Galactic disk. This provides clues of the launching point of the PA, roughly $6 \times 10^{6}$ years ago. Implications of the dynamical time scale of the PA might be related to the Galactic Center Lobe (GCL) at parsec scale. Our results suggest that in the central 30 pc of the GC, the feedback from past explosions could alter the orbital path of the molecular gas down to the central tenth of parsec. In the follow-up work of our new $\operatorname{CS}(J=2-1)$ map, we also find that near the systemic velocity, the molecular gas shows an extraplanar hourglass-shaped feature (HG-feature) with a size of $\sim 13$ pc. The latitude-velocity diagrams show that the eastern edge of the HG-feature is associated with an expanding bubble B1, $\sim 7$ pc away from SgrA*. The dynamical time scale of this bubble is $\sim 3 \times 10^{5}$ years. This bubble is interacting with the $50 \mathrm{~km} \mathrm{~s}^{-1}$ cloud. Part of the molecular gas from the $50 \mathrm{~km}$ $\mathrm{s}^{-1}$ cloud was swept away by the bubble to $b=-0.2^{\circ}$. The western edge of the HG-feature seems to be the molecular gas entrained from the $20 \mathrm{~km} \mathrm{~s}^{-1}$ cloud towards the north of the Galactic disk. Our results suggest a fossil explosion in the central $30 \mathrm{pc}$ of the GC a few $10^{5}$ years ago.
\end{abstract}

Subject headings: Galaxy: center - radio lines: ISM - ISM: molecules - Galaxy: structure - techniques: image processing 


\section{INTRODUCTION}

Our Galactic Center (GC) is the nearest nucleus of a galaxy ( $d=8.5 \mathrm{kpc}$ ) (Reid 1993; Ghez et al. 2008; Reid et al. 2014). It is the best target to study detailed structures and dynamics in a circumnuclear environment at sub-pc scale, which cannot be easily done in external galaxies with ground-based telescopes. However, observations of the GC suffer from our edge-on vantage point. Optical and near infrared (IR) emission suffers from large amounts of extinction. The V-band extinction $\left(A_{\mathrm{v}}\right)$ varies from 20 to 50 mag with a median value of $31.1 \mathrm{mag}$ (Scoville et al. 2003). Dust is transparent at radio wavelengths. However, at millimeter wavelengths, the more abundant molecular lines (e.g., CO, $\mathrm{J}=1-0$ ) become optically thick and suffer from foreground absorption or self-absorption in the direction towards the GC (Guesten et al. 1987; Wright et al. 2001a; Christopher et al. 2005a). High-excitation molecular lines, or high-density tracers are less affected by the foreground/ambient cold gas (Montero-Castaño et al.|2009; Tsuboi et al. 1999; Jackson et al. 1993; McGary et al. 2001; McGary \& Ho 2002; Herrnstein \& Ho 2002, $2005)$.

Fruitful studies were already carried out with low-excitation molecular lines and with low angular resolutions (e.g. Scoville 1972; Burton \& Liszt 1983; Bally et al. 1987, 1988; Burton \& Liszt 1992; Tsuboi et al. 1999). Our focus is on the complex activities and physical conditions in the nuclear region probed by the dense molecular gas. We conducted wide-field single-dish observations of the central $30 \mathrm{pc}$ of the GC with multiple transitions of the CS molecule (Hsieh et al. 2015, hereafter paper I). In paper I, we reported a new feature called the connecting ridge $(\mathrm{CR})$ which was detected with the $\mathrm{CS}(J=4-3)$ line. The $\mathrm{CR}$ has a velocity gradient perpendicular to the disk rotation. It is physically associated with the extraplanar polar arc (PA) (Bally et al. 1988; Henshaw et al. 2016). The PA extends from north of the $\mathrm{SgrA}^{*}$ region at a $40^{\circ}$ angle and shows a large velocity gradient from 
$\left(l, b, V_{\text {sys }}\right)=\left(0^{\circ}, 0.05,70 \mathrm{~km} \mathrm{~s}^{-1}\right)$ to $\left(0.2,0.25,140 \mathrm{~km} \mathrm{~s}^{-1}\right)$. Below $V_{\text {sys }}$ of $70 \mathrm{~km} \mathrm{~s}^{-1}$, the PA lies close to the Galactic plane and becomes confused with the molecular clouds in the $\mathrm{Sgr}^{*}$ region. In paper I, we find that the kinematic and spatial structures connect the Galactic disk, the CR, and the PA. These results suggest that the molecular gas might be lifted out of the Galactic plane. We, thus, proposed the idea of a molecular outflow in the central $30 \mathrm{pc}$ of the GC and suggested that the PA is pushed away, possibly by the energy of 8-80 supernovae explosions. The importance of Galactic outflows is that they may be the primary mechanism to recycle metals and to deposit them into the intergalactic medium (Veilleux et al. 2005). In starburst galaxies, vast amounts of stellar winds from massive stars as well as supernovae explosions generate a huge amount of energy and high pressure to create high-velocity galactic winds, which interact with and sweep up the ambient gas. The GC is the nearest nucleus, and therefore, it is the best target to resolve the structure, kinematics, and physical conditions of nuclear outflows. The presence of atomic and molecular gas allows us to measure the outflow of neutral material, its impact, and transfer of energy and momentum to the surrounding interstellar medium (ISM). In this paper, we continue to investigate the possibility of the outflow nature in the GC with our new $\operatorname{CS}(J=2-1)$ data.

\section{OBSERVATIONS AND DATA REDUCTION}

The central molecular zone (CMZ) of the Milky Way was observed in the CS(J = 2-1; $97.98093 \mathrm{GHz}$ ) line with the $45 \mathrm{~m}$ telescope of the Nobeyama Radio Observatory (NRO) 1 in May 2012. The full width at half maximum (FWHM) of the beam size at this frequency was

\footnotetext{
${ }^{1}$ Nobeyama Radio Observatory is a branch of the National Astronomical Observatory of Japan, National Institutes of Natural Sciences.
} 
$\sim 17^{\prime \prime}$. We used the 25 elements of the focal plane receiver (BEam Array Receiver System: BEARS) (Sunada et al. 2000) to observe the central $108^{\prime} \times 27^{\prime}(l \times b)$ area of the CMZ in the On-The-Fly (OTF) mapping mode (Sawada et al. 2008) with position-switching. As the backend spectrometer we used digital auto-correlators with a bandwidth of $512 \mathrm{MHz}$ with a spectral resolution of $500 \mathrm{kHz}$ with 1024 channels. The total bandwidth was $\sim 1740$ $\mathrm{km} \mathrm{s}^{-1}$ with a resolution of $1.3 \mathrm{~km} \mathrm{~s}^{-1}$ in the raw data.

The mapping area was arranged into four sub-regions, each covering $27^{\prime} \times 27^{\prime}$. We sampled an emission-free reference $(\mathrm{OFF})$ position at $(l, b)=\left(1 .^{\circ},-0.7^{\circ}\right)$ for every two 35-second on-source scans along each sub-region. The sampling interval along the scan rows was $5 \prime \prime 1$, and the separation between the scan rows was 7.5 , which corresponds to roughly $1 / 3$ and $1 / 2$ of the beam. We scanned along the $l$ and $b$ directions in order to minimize scanning artefacts with the basket-weave method (Emerson \& Graeve 1988). These artefacts originate likely from pointing errors, time variations of the system temperature $T_{\text {sys }}$, insufficient sampling grids, and the non-uniform beam separations of the BEARS elements (Sawada et al. 2008).

The pointing of the antenna was corrected by measuring the SiO maser VX Sgr every hour with the SIS receiver S40 in the 40-GHz band. The Double Side Band (DSB) $T_{\text {sys }}$ varied from 300 to $700 \mathrm{~K}$ during our observations. We used the standard chopper wheel method to calibrate the output signal into the antenna temperature $T_{\mathrm{A}}^{*}$, which was corrected for atmospheric attenuation. Since the original $T_{\text {sys }}$ measured by BEARS were DSB $T_{\text {sys }}$, we needed to measure the relative gains of the two sidebands. We used the single beam receiver S100, equipped with a Single Side Band (SSB) filter to observe a calibrator. Every beam of BEARS was then scaled to convert from $T_{\mathrm{A}}^{*}(\mathrm{DSB})$ to $T_{\mathrm{A}}^{*}(\mathrm{SSB})$. The main beam efficiency $\eta_{\mathrm{MB}}$ was $\sim 43 \%$. In the maps presented here we are using the $T_{\mathrm{A}}^{*}(\mathrm{SSB})$ scale.

We used the NOSTAR package (Sawada et al. 2008) to reduce our OTF data. The 
baselines were subtracted with linear or higher-order polynomial functions, and bad scans were flagged. During the observations, one of the receivers (A09) had abnormally high $T_{\text {sys }}$. Therefore, the data of A09 were flagged, and we only used 24 receivers. The resulting gridding size of the map is $7.5^{\prime \prime}$, with an effective final resolution of $21^{\prime \prime}$ with bessel-gaussian convolution and an rms noise of $0.1 \mathrm{~K}\left(T_{\mathrm{A}}^{*}\right)$ at a velocity resolution of $2.5 \mathrm{~km} \mathrm{~s}^{-1}$. In this paper, we present the $\operatorname{CS}(J=2-1)$ line data for the central $30^{\prime}$ (70 pc) region of Milky Way. The entire $\operatorname{CS}(J=2-1)$ data of the CMZ will be presented in another paper.

\section{The Central 30 pc of the Milky Way}

\section{1. $\quad \operatorname{CS}(J=2-1)$ Line Map}

In Figure 1 we show the $\operatorname{CS}(J=2-1)$ and $\operatorname{CS}(J=1-0)$ (Tsuboi et al. 1999) integrated intensities. We overlay the $\operatorname{CS}(J=2-1)$ channel maps on the VLA archival 20-cm radio continuum emission in Figure 2. The noise levels of these two data sets are comparable. The integrated intensity maps of both transitions are smoothed to $30^{\prime \prime}(\sim 1 \mathrm{pc})$ for comparison. In general, the structures are consistent in both transitions but the new $\mathrm{CS}(J=2-1)$ line map reveals more details and extended emission than the $\operatorname{CS}(J=1-0)$ line. This is due to the high excitation properties of the GC (e.g. Morris \& Serabyn 1996; Montero-Castaño et al. 2009). Two molecular clouds, called $20 \mathrm{~km} \mathrm{~s}^{-1}$ cloud (hereafter $20 \mathrm{MC}$ ) and $50 \mathrm{~km} \mathrm{~s}^{-1}$ cloud (hereafter $50 \mathrm{MC}$ ), are well studied in the GC (e.g., Guesten et al. 1981; Tsuboi et al. 2009; Liu et al. 2012). These two clouds are part of the central molecular zone (CMZ) (e.g., Morris \& Serabyn 1996). The $50 \mathrm{MC}$ is known to interact with the SNR SgrA East (e.g., Serabvn et al. 1992), and the $20 \mathrm{MC}$ is feeding the circumnuclear disk (CND) (e.g., Ho et al. 1985), which is a ring-like feature in between the 20/50 MC (e.g., Guesten et al. 1987; Jackson et al. 1993; Amo-Baladrón et al. 2011; Harris et al. 1985; Mezger et al. 1989; Etxaluze et al. 2011; Lau et al. 2013; Wright et al. 
2001b; Montero-Castaño et al. 2009; Martín et al. 2012; Herrnstein \& Ho 2002, 2005;

Christopher et al. 2005b; Requena-Torres et al. 2012; Mills et al. 2013). The CND shows a fast rotation from $-120 \mathrm{~km} \mathrm{~s}^{-1}$ to $120 \mathrm{~km} \mathrm{~s}^{-1}$. It is known to have a high-excitation state and is, thus, more clearly seen in $\operatorname{CS}(J=2-1)$ than in $\operatorname{CS}(J=1-0)$ (e.g., McGary et al. 2001; McGary \& Ho 2002; Montero-Castaño et al. 2009). The polar arc (PA) (Bally et al. 1988) is a high-velocity cloud with velocities up to $\geq 100 \mathrm{~km} \mathrm{~s}^{-1}$. It shows a high gas density (Tsuboi et al. 1999) but its origin is not clear. In paper I, we reported that the PA appears to be physically connected to the Galactic disk and it may originate from the disk. The PA is also the molecular counterpart of the eastern spur of the Galactic Center Lobe (GCL) (e.g., Sofue 1996). The CS( $J=2-1)$ line emission located at the base of the PA appears both at the negative velocity of $-50 \mathrm{~km} \mathrm{~s}^{-1}$ and at the positive velocities of 60 to $80 \mathrm{~km} \mathrm{~s}^{-1}$. This double-peak feature is called the molecular loop (ML) in paper I.

In Figure 2, we note that the $\operatorname{CS}(J=2-1)$ emission seems to have an interesting spatial association with the 20 -cm radio continuum. In particular, the molecular gas around $-20 \mathrm{~km} \mathrm{~s}^{-1}$ to $20 \mathrm{~km} \mathrm{~s}^{-1}$ shows an hourglass-shaped feature (hereafter HG-feature), with a northwest-southeast orientation perpendicular to the Galactic disk, with the openings or cavities that surround the radio halo (Pedlar et al. 1989; Zhao et al. 2014). In the negative Galactic plane, the 20/50 MC seem to surround the southern radio halo. Above SgrA*, there is also some molecular gas which coincides with the filaments inside the radio lobe. We compare this with the previous $\operatorname{CS}(J=1-0)$ line map (Tsuboi et al. 1999). To avoid any possible contamination from the 20/50 MC, we show in Figure 3 the intensity map integrated for $\pm 10 \mathrm{~km} \mathrm{~s}^{-1}$ based on higher-resolution channel maps (2.5 $\mathrm{km} \mathrm{s}^{-1}$ resolution). We overlay the CS line maps on the 20-cm continuum map to show more clearly the HG-feature structures. In general, $\operatorname{CS}(J=2-1)$ and $\operatorname{CS}(J=1-0)$ have a similar morphology. With a comparable sensitivity, our new $\operatorname{CS}(J=2-1)$ line emission shows a more extended structure than $\operatorname{CS}(J=1-0)$. 
In Figure 4, we display the color-composite map of the 20-cm (Yusef-Zadeh et al. 2004) and the MSX E-band 21- $\mu \mathrm{m}$ map (Price et al. 2001; Simpson et al. 1999, 2007). We compare the $\operatorname{CS}(J=2-1)$ line emission with the dust $(21 \mu \mathrm{m})$ and the emission from free electrons $(20 \mathrm{~cm})$. The map shows that the low-velocity gas roughly surrounds the infrared/radio features. The $21-\mu \mathrm{m}$ emission shows the prominent Galactic center bubble (Simpson et al. 2007) (GC bubble, or arc bubble in Ponti et al. 2015) near the SgrA radio halo/arc. The northern part of the GC bubble has a radio feature known as the "radio arc" (e.g. Serabyn \& Guesten 1987; Lang et al. 1999; Yusef-Zadeh et al.|1984). The GC bubble could be produced by the starbursts in the past (Simpson et al. 2007; Sofue 2003). Our $\operatorname{CS}(J=2-1)$ line map spatially coincides with the infrared/radio features. The HG-feature seems to surround the warm dust and the free-electron emission.

In summary, our new high-resolution/excitation $\operatorname{CS}(J=2-1)$ line map suggests that the motions in the central $30 \mathrm{pc}$ are not simply rotation but are also associated with structures in the vertical direction of the Galactic plane. In the following, we will revisit the association of the known features with the HG-feature and the PA.

\subsection{Latitude-Velocity Diagrams}

Latitude-velocity diagrams $(b-v$ diagram) of the CS line data are presented to investigate the extraplanar kinematics. Figure 5 specifies the range for the $b-v$ diagrams. The $b-v$ diagrams are presented for both $\operatorname{CS}(J=2-1)$ and $\operatorname{CS}(J=1-0)$ data at a resolution of $30^{\prime \prime} \times 5 \mathrm{~km} \mathrm{~s}^{-1}$ (Figure 6 to Figure 11). In order to improve the signal-to-noise ratios, the $b-v$ diagrams are averaged every $2.6^{\prime}$ in longitude $(\Delta l)$ from $l=0.07^{\circ}$ to $l=-0.18^{\circ}$ from Figure 7 to Figure 11, The gaps between regions are $30^{\prime \prime}$, which corresponds to the convolved beam size. As shown in Figure 5, region A and region B present the eastern edge of the HG-feature. Region C covers SgrA* and the CND. Region D and Region 
E cover the western edge of the HG-feature. From a visual inspection, there are several apparent features in the $b-v$ diagrams. In Figure 6, we also present the $b-v$ diagram averaged over the entire $\mathrm{HG}$-feature (from $l=0.07^{\circ}$ to $l=-0.18^{\circ}$ ) (Region A to E). In the following, we briefly summarize the known/new features with our new wide-field data in this complicated region.

1. The 20 MC and the 50 MC: In Figure 6, the 20/50 MC appear south of the Galactic plane from $b=-0.02^{\circ}$ to $b=-0.14^{\circ}$ with velocities from $\sim 80 \mathrm{~km} \mathrm{~s}^{-1}$ to $\sim-30 \mathrm{~km} \mathrm{~s}^{-1}$. These two molecular clouds are well studied and are known to be located in the Galactic disk (e.g., Guesten et al. 1981; Coil \& Ho 2000; McGary et al. 2001; Serabyn et al. 1992; Liu et al. 2012). The morphology of these two clouds is consistent in both CS lines.

The $50 \mathrm{MC}$ appears in the regions A, B, and C (Figure 7, 8, 9). Tsuboi et al. (2009) discussed the expanding properties of the central 50 MC (Region B). A SNR candidate is located in the expanding shell in the $50 \mathrm{MC}$ (Tsuboi et al. 2009). The 20 MC appears in the regions D and E. (Figure 10, 11). A SNR candidate, G359.92-0.09 (wisp; Ho et al. 1985, $l=359.89^{\circ}, b=-0.086^{\circ}$ ), in the Galactic center region was studied by the ASCA and the Chandra telescopes (Senda et al. 2003). The interaction of the wisp and the $20 \mathrm{MC}$ was also investigated by Ho et al. (1985) and Coil \& Ho $(2000)$.

2. The Circumnuclear Disk (CND): The CND appears in the regions C and D (Figure 9, 10) in $\operatorname{CS}(J=2-1)$. As shown in the channel maps. it has high velocities of up to $\pm 120 \mathrm{~km} \mathrm{~s}^{-1}$ and a steep velocity gradient as compared to the Galactic disk. The CND is known to have high excitation (Mills et al. 2013; Montero-Castaño et al. 2009). This explains why the CND appears more significant in $\operatorname{CS}(J=2-1)$ than in $\mathrm{CS}(J=1-0)$ 
3. The High-Velocity Compact Cloud (HVCC) CO 0.02-0.02: In region B, there is a high-velocity clump $\left(b=-0.015^{\circ} ; v \sim 90 \mathrm{~km} \mathrm{~s}^{-1}\right)$ with a broad linewidth $\geq 100$ $\mathrm{km} \mathrm{s}^{-1}$ and with sizes of $\sim 3-4 \mathrm{pc}$. This is one of the HVCCs studied by Oka et al. (1999, 2008) (CO 0.02-0.02). This HVCC is located in the "finger-like" ridge that connects to the CND. This HVCC was suggested to be impacted upon by the SN explosions resulting in an unusually high density and temperature (Oka et al. 1999, 2008, 2011).

4. The Polar Arc (PA): The PA represents a pair of arcs with positive and negative components above the Galactic plane (green dashed lines, Figure 6 to Figure 11) that extend down to $b \leq 0.23^{\circ}$ and become confused with the Galactic disk. A velocity gradient of $\sim 5.5 \mathrm{~km} \mathrm{~s}^{-1} \mathrm{pc}^{-1}$ is seen across the PA. The ML mentioned in Sect. 3.1 is located at the contact point of the PA and the Galactic disk around $b=0.03^{\circ}$. Velocities of the ML at the intensity peaks are $\sim 60 \mathrm{~km} \mathrm{~s}^{-1}$ and $\sim-50 \mathrm{~km} \mathrm{~s}^{-1}$. The ML and the PA appear from region A to region E with a spatial length of $\sim 34$ pc. The ML seems to be part of the PA in the $b-v$ diagrams based on their coherent locations and kinematics. The $\operatorname{CS}(J=4-3) \mathrm{CR}$ studied in paper I fills the gap between the positive-velocity knot of the ML and the Galactic disk. In addition, the positive-velocity component is brighter than the negative-velocity component by a factor of $\sim 1.5$. The $b-v$ diagrams of the PA and the ML indicate an accelerating and expanding motion perpendicular to the Galactic disk. Similar kinematic structures are seen in outflows from starburst galaxies (e.g., Walter et al. 2002; Tsai et al. 2009; Bolatto et al. 2013) but on kilo-pc scales. It is particularly important to note that all the emission north of the Galactic plane, with positive and negative velocities, converges in the $b-v$ diagrams towards $\operatorname{SgrA}^{*}$ in the nucleus of the Galaxy in our high-resolution map. 
5. The Newly Found Bubble?: In Figure 6, the low-level emission as indicated by the red ellipse in the southern Galactic plane shows a half-curve feature with a central cavity (here, we call this feature B1). The newly found B1 appears in the regions $\mathrm{A}, \mathrm{B}, \mathrm{C}$ and seems to extend to the northern Galactic plane marked by the blue ellipse, called B2 in Figure 7. The kinematics of the B1 can be explained by an expanding bubble in the $b-v$ diagrams. Above $b=-0.1^{\circ}$, the B1 is blended with the $50 \mathrm{MC}$ and the $-13 \mathrm{~km} \mathrm{~s}^{-1}$ cloud. The structure inside the Galactic disk is very complicated, and it is not clear how to identify the boundary of the northern side of either B1 or B2. For a visual inspection, the center and the radius of B1 are $(b, v)=\left(-0.098^{\circ}, 19 \mathrm{~km} \mathrm{~s}^{-1}\right)$ and $(\Delta b, \Delta v)=\left(0.1^{\circ}, 40 \mathrm{~km} \mathrm{~s}^{-1}\right)$, respectively. From Figure 7 to Figure 9, some gas in the 50 MC shows a smooth connection to the B1 from $40 \mathrm{~km} \mathrm{~s}^{-1}$ to $70 \mathrm{~km} \mathrm{~s}^{-1}$. To avoid any contamination from the CND, we present intensity ratio maps by averaging region A and B (Figure 12). We exclude ratios lower than $3 \sigma$ and hence, the emission in the southern Galactic plane is discarded due to its low-level emission. High ratios $\geq 3.5$ appear in the HVCC CO0.02-0.02 and also in the $\mathrm{B} 2$. At the contact point of the $50 \mathrm{MC}$ and the $\mathrm{B} 1$, the ratios are also $\geq 3.5$. In general, the remaining disk emission has ratios lower than 2.

6. The Hourglass-Shaped (HG) Feature: We have identified the HG-feature in the low-velocity channels (Figure 2). Here, we investigate the kinematics of this structure in the $b-v$ diagrams. We are specifically examining the extraplanar nature of this feature. The low-velocity structure of the HG-feature is labeled as yellow box from Figure 7 to Figure [1], where the regions A/B and D/E trace the eastern and western edges, respectively. The eastern edge of the HG-feature (Figure 7) corresponds to the blueshifted side of the B1. This side is not blended with the $50 \mathrm{MC}$ and $20 \mathrm{MC}$, and hence can be seen as a half-shell feature. The western edge of the HG-feature shows a half-arc feature and seems to smoothly connect to the $20 \mathrm{MC}$ out to the 
northern plane (western HG) with increasing velocity. The western HG is mostly located between $-10 \mathrm{~km} \mathrm{~s}^{-1}$ and $10 \mathrm{~km} \mathrm{~s}^{-1}$. We also present the intensity ratio map generated by averaging region D and E in Figure 12. The high-ratio ( $\geq 3)$ data concentrate on the western HG.

\section{Polar Arc: Galactic Center Molecular Outflow?}

In the $b-v$ diagrams (Figure 6 ), we find that the velocity of the PA is linearly increasing along the Galactic latitude to more than $100 \mathrm{~km} \mathrm{~s}^{-1}$. This can be interpreted as an expanding motion. Similar features caused by kpc-scale molecular outflows are seen in the nearby starburst galaxies, e.g., NGC 2146, NGC 3628, NGC 253, M82 (Tsai et al. 2009; Bolatto et al. 2013; García-Burillo et al. 2001; Walter et al. 2002). These molecular outflows are suggested to be the late evolved stage of superbubbles (Yokoo et al. 1993), which are driven by intensive stellar winds and supernovae explosions (Veilleux et al. 2005). The velocities of the GC outflow are typical values of molecular outflows seen in external starburst galaxies ( $100 \mathrm{~km} \mathrm{~s}^{-1}$ on average; Veilleux 2004), which are generally lower than the velocities of their ionized-gas outflows. With the implications of (1) extraplanar structures, and (2) expanding/accelerating motions, we suggest that the polar arc is associated with the molecular outflow. We then estimate the kinetic properties of the outflow by assuming a constant acceleration. For the positive-velocity ridge, we use a velocity of $\sim 100 \mathrm{~km} \mathrm{~s}^{-1}$ and a length of $34 \mathrm{pc}$ (as shown in Figure 6), which then gives an acceleration $a_{\mathrm{pos}}=0.5 \times\left(100 \mathrm{~km} \mathrm{~s}^{-1}\right)^{2} /(34 \mathrm{pc})=4.8 \times 10^{-12} \mathrm{~km} \mathrm{~s}^{-2}$, and a dynamical time scale $t_{\text {pos }}=\left(100 \mathrm{~km} \mathrm{~s}^{-1}\right) / 4.8 \times 10^{-12} \mathrm{~km} \mathrm{~s}^{-2}=6.7 \times 10^{5}$ years. We note that this value is averaged over the structure. As shown in paper I, the PA belongs to the eastern protrusion of the GCL (see Figure 20 in paper I). The time scale of the PA is close to the dynamical time scale of the GCL (1 Myr) (Bland-Hawthorn \& Cohen 2003). Implications 
of the extraplanar PA can be traced back to the disk region (the ML), providing clues of the launching point of the PA, and perhaps a link to the GCL at parsec scale. At kpc-scale, fossil imprints of past explosions in the GC were reported in Bland-Hawthorn \& Cohen (e.g., 2003); Su et al. (e.g., 2010); Crocker et al. (e.g., 2011). For example, there is evidence that the GC experienced episodic starbursts a few Myr ago, producing more than 100 SNe during the entire starburst (q.v. Tamblvn \& Rieke 1993; Siouwerman et al. 1998; Simpson et al. 1999; Hartmann 1995; Ozernoy 1996; Carretti et al. 2013). Detailed modeling of the low-excitation fine-structure line spectrum (Lutz 1999) suggests a starburst event about 7 Myr ago. It is also suggested that SgrA* went through a Seyfert phase in the recent past (q.v. Zubovas et al. 2011; Guo \& Mathews 2012; Bland-Hawthorn et al. 2013). Guo \& Mathews (2012) propose that the SgrA* jets, which inflated the Fermi bubble ( $\mathrm{Su}$ et al. 2010), formed $1-3 \mathrm{Myr}$ ago and persisted for $0.1-0.5 \mathrm{Myr}$. The total energy of the $\mathrm{SgrA}^{*}$ jets is in the range of $10^{55-57} \mathrm{erg}$. The $A S C A 2-10 \mathrm{keV}$ observations of the CMZ also provide evidence that $\operatorname{SgrA}^{*}$ was $10^{5}$ times more active in the past $10^{3}$ years. The fluorescent X-ray emission from cold iron atoms in the molecular clouds of the CMZ is possibly due to the X-ray irradiation from SgrA* flares (q.v. Sunyaev et al. 1993; Koyama et al. 1996; Ponti et al. 2010). Bland-Hawthorn et al. (2013) also suggest that the $\mathrm{H} \alpha$ emission of the Magellanic Stream arose from an accretion flare of SgrA* $1-3$ Myr ago. The required star-formation rate to produce the $\mathrm{H} \alpha$ emission of the Magellanic stream is at least two orders of magnitude larger than what can be generated by the star formation history of the GC. In this paper, we have confirmed that the PA originates from the Galactic disk. Henshaw et al. (2016) also show that the PA seems to extend from Arm I (Sofue 1995), but with an offset in velocity. Our results suggest that in the central 30 pc of the GC, the feedback from past explosions could alter the orbital path of the molecular gas down to the central tenth of parsec. 


\section{Extraplanar Features in the Galactic Center}

In the $b-v$ diagrams (Figure 6), the molecular feature B1 appearing below $b=-0.1^{\circ}$ suggests an expanding bubble. The B1 has higher $\operatorname{CS}(J=2-1) / \operatorname{CS}(J=1-0)$ line ratios $(\sim 3)$ than the ambient $20 / 50 \mathrm{MC}$ by a factor of 2 . The B1 has a counterpart in

$\mathrm{SiO}(J=2-1)$ (Tsuboi et al. 2011) suggesting that the low-level emission of B1 is shocked. At $v=-20$ to $v=60 \mathrm{~km} \mathrm{~s}^{-1}$, the B1 seems to interact with the $20 / 50 \mathrm{MC}$, as these clouds curve around the entire southern part of the shell in the $b-v$ diagrams (marked by the ellipse of B1). The northern boundary $\left(b \geq-0.1^{\circ}\right)$ of the B1 is not clear due to the complicated structures inside the 20/50 MC. It is possible that the B1 extends to the 20/50 MC as hinted in the ratio map, marked as B2 (Figure 12). The B2 has ratios higher than 3 and shows a shell-like feature in the 20/50 MC. In region A (Figure [7), above $b=0^{\circ}$, the "low-velocity" emission tracing the eastern edge of the HG-feature (yellow box) is hard to identify because of the confusion of the Galactic disk. This "low-velocity" emission might also belong to the "high-velocity" part of the B1 if we consider that the B1 extends up to $b=0.05^{\circ}$. In this sense, the eastern edge of the HG-shaped feature might be part of the B1. In any case, we estimate the lower limit of the kinetic parameters with the B1. With an expanding velocity of $\sim 40 \mathrm{~km} \mathrm{~s}^{-1}$, the kinetic energy of the northern HG-feature is $\frac{1}{2} M_{\text {shell }} V_{\text {shell }}^{2}$, which is at the order of $\sim 10^{49}$ erg with an $\mathrm{H}_{2}$ mass of $\sim 1000 M_{\odot}$. The $\mathrm{H}_{2}$ mass is adopted from a Local Thermal Equilibrium (LTE) condition, with an excitation temperature of $20 \mathrm{~K}$ (paper I), a CS abundance of $5 \times 10^{-9}$ (Martín et al. 2008), and the measured brightness temperature from the $\operatorname{CS}(J=1-0)$ line. The shell kinetic energy could be different by a factor of 0.1 to 10 for different CS abundances and excitation temperatures. The expansion time of the B1 is $\sim 3 \times 10^{5}$ years with the above expansion velocity of $40 \mathrm{~km} \mathrm{~s}^{-1}$ and a size of $13 \mathrm{pc}$. We notice that the time scales of the B1 and the PA are similar. The time scale is larger than the age of SgrA East (a few $10^{4}$ yr; e.g., Herrnstein \& Ho 2005). However, we note that the current measured expansion velocity sets 
an upper limit on the age, because the expansion speed was most likely larger in the past. Nevertheless, the identification of these individual shells is not unique or certain. There is also the possibility that all of the features are related in that they may be complexities on top of a general outflowing shell.

The western edge of the HG-feature (western-HG) is located in the region D (Figure 10). There is a velocity continuation of the $20 \mathrm{MC}$ above SgrA*. This continuation traces the western edge of the HG-feature above the Galactic plane from $(b, v)=\left(-0.03^{\circ},-10 \mathrm{~km} \mathrm{~s}^{-1}\right)$ to $(b, v)=\left(0.06^{\circ}, 30 \mathrm{~km} \mathrm{~s}^{-1}\right)$. The velocity of this continuation is increasing out to the Galactic latitude and corresponds to the western-HG. Rather than a closed expanding bubble, this feature seems to be entrained and swept into the HG-feature from the $20 \mathrm{MC}$, which shows a similar kinematic behavior as the PA. The ratios $(\geq 4.5)$ of the western-HG are higher than those of the cold disk emission $(\leq 2.5)$, which suggests a higher excitation state in the western-HG. However, the structures in this region are complicated, and it is difficult to identify structures in the $b-v$ diagrams. Moreover, the current results do not show evidence that the HG-feature is a physical bipolar feature because the western and eastern edges do not show a coherent shell expansion. The asymmetry of the structures in the $b-v$ diagrams might suggest that the outflow phenomenon must be greatly influenced by the asymmetric circumnuclear environment.

In the following, we discuss the physical association between the HG-feature and the radio halo/lobe. The bipolar radio lobe has counterparts in X-ray emission (Maeda et al. 2002; Markoff 2010). Mixtures of the thermal S and Si emission in the radio lobe suggest a thermal origin of the hot plasma (Park et al. 2004), heated by the shocks from SNe and massive stellar winds. The interaction of the hot plasma with the ambient dense cloud might also produce the observed neutral Fe line. It is unclear whether the hot plasma from the radio lobe in situ can entrain the molecular gas. We do a self-consistent check 
of whether the ionized gas of the bipolar radio-lobe is able to entrain the molecular gas from the disk clouds, assuming it is moving outward perpendicular to the GC. For the given emission measure of $2.7 \times 10^{5} \mathrm{pc} \mathrm{cm}^{-6}$ and the source size of $4^{\prime}$ (Pedlar et al. 1989), we derive the electron density $n_{\mathrm{e}}$ to be $7.8 \times 10^{4} \mathrm{~cm}^{-3}$. The electron temperature is $T_{\mathrm{e}}=$ $5000 \mathrm{~K}$ adapted from Pedlar et al. (1989). Therefore, we estimate the thermal ionized gas pressure to be $P_{\mathrm{i}} / k=n_{\mathrm{e}} T_{\mathrm{e}} \sim 4 \times 10^{8} \mathrm{~cm}^{-3} \mathrm{~K}$. The thermal pressure of the molecular gas is derived from $P_{\mathrm{m}} / k=n_{\mathrm{H}_{2}} T_{\text {kin }}$, where $P_{\mathrm{m}} / k$ is the gas thermal pressure, $n_{\mathrm{H}_{2}}$ is the number density of the molecular gas, and $T_{\text {kin }}$ is the kinetic temperature. We assume $n_{\mathrm{H}_{2}}$ is $10^{5}$ $\mathrm{cm}^{-3}$ (the critical density of $\operatorname{CS}(J=2-1)$ ), and a kinetic temperature of $20 \mathrm{~K}$ (paper I). The $P_{\mathrm{m}} / k$ is then derived as $2 \times 10^{6} \mathrm{~cm}^{-3} \mathrm{~K}\left(P_{\mathrm{m}}=7 \times 10^{-10} \mathrm{erg} \mathrm{cm}^{-3}\right)$. The thermal pressure of the ionized lobe is, thus, sufficient to entrain the molecular gas. An alternative way is to measure the momentum of the radio halo and compare it with the molecular gas (e.g., Matsushita et al. 2004, 2007). However, we are currently lacking velocity information of the radio halo. Future kinematic measurements of the radio halo will help to further elucidate the interaction between the ionized and molecular gas.

\section{SUMMARY}

From our 1-pc resolution $\operatorname{CS}(J=2-1)$ line maps, we find that the molecular gas in the central $30 \mathrm{pc}$ of the Galactic Center has a morphology that is reflecting recent explosions $(3-6) \times 10^{5}$ years ago. As a follow-up study of paper I, we revisit the idea that the PA is a molecular outflow launched from the Galactic disk. The linearly increasing velocity of the PA suggests that it is moving out of the Galactic disk. Near the systematic velocity, the molecular gas shows an HG-feature, which might be a mixture of multiple explosive events. The north-western edge of the HG-feature might trace the entrainment from the $20 \mathrm{MC}$ from $b=-0.07^{\circ}$ to $b=0.06^{\circ}$. The southern part of the eastern edge of 
the HG-feature suggests an expanding bubble (B1). The low-level emission of B1 south to the Galactic plane is shocked. These individual features might be complexities on top of a general outflowing shell.

We thank the reviewer for a thoughtful review and suggestions to improve our manuscript. We thank Dr. Yusef-Zadeh for providing us with the VLA $20 \mathrm{~cm}$ continuum map. Hsieh, Pei-Ying is supported by the National Science Council (NSC) and the Ministry of Science and Technology (MoST) of Taiwan through the grants NSC 100-2112-M-001-006MY3, NSC 97-2112-M-001-021-MY3, and MoST 103-2112-M-001-032-MY3.

This research made use of data products from the Midcourse Space Experiment. This research has also made use of the NASA/ IPAC Infrared Science Archive, which is operated by the Jet Propulsion Laboratory, California Institute of Technology, under contract with the National Aeronautics and Space Administration. 

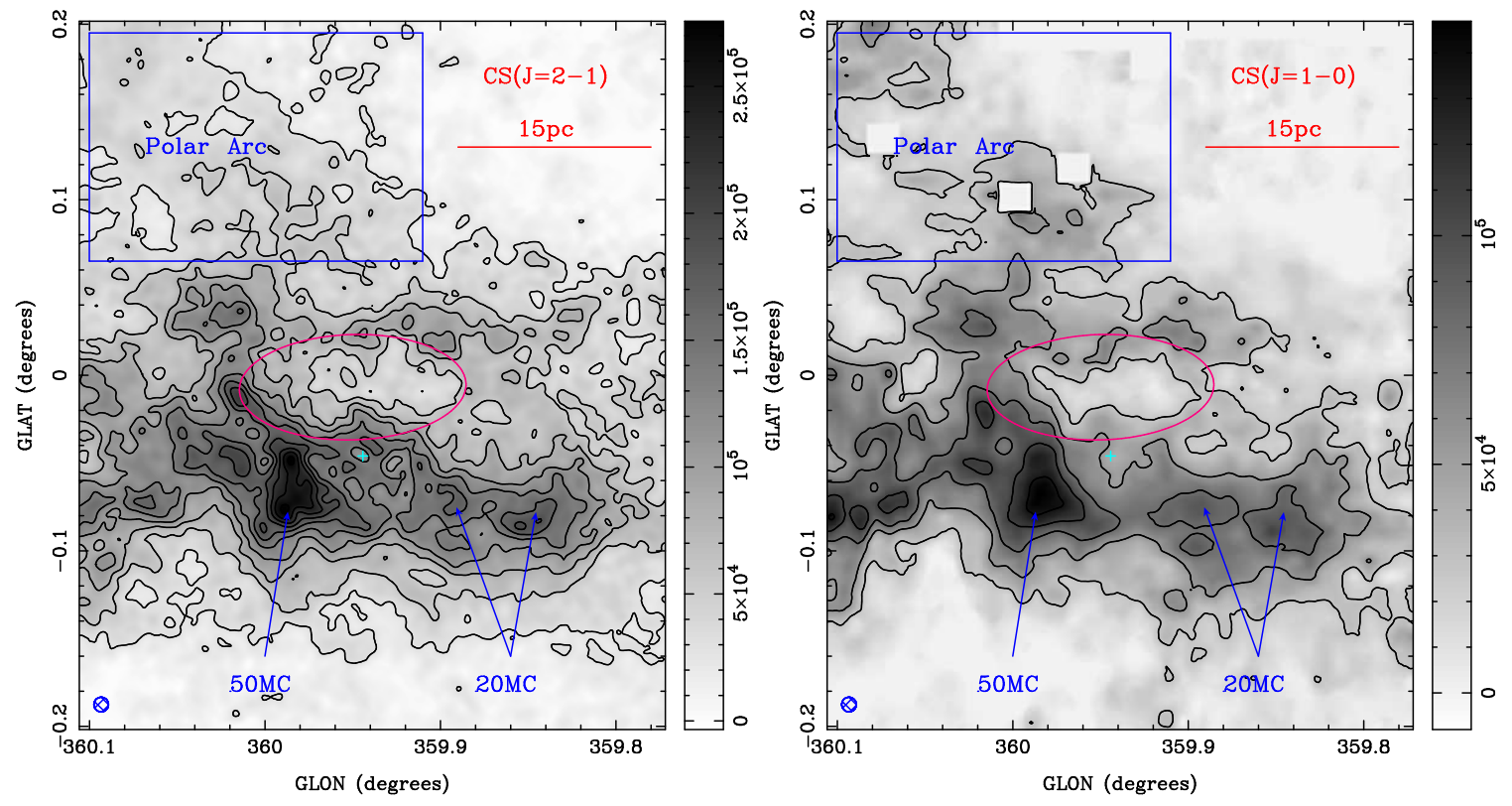

Fig. 1.- Top: $\operatorname{CS}(J=2-1)$ emission integrated from $-197.5 \mathrm{~km} \mathrm{~s}^{-1}$ to $197.5 \mathrm{~km} \mathrm{~s}^{-1}$ ( $T_{\mathrm{A} *}$ in unit of $\mathrm{K} \mathrm{m} \mathrm{s}^{-1}$ ). Bottom: $\mathrm{CS}(J=1-0)$ line emission (Tsuboi et al. 1999) integrated from $-197.5 \mathrm{~km}$ $\mathrm{s}^{-1}$ to $197.5 \mathrm{~km} \mathrm{~s}^{-1}$. The beam size of the $\operatorname{CS}(J=2-1)$ and $\operatorname{CS}(J=1-0)$ line maps is $30^{\prime \prime}$ (blue circles in lower left corners). The contours are $5,10,15, \ldots, 50 \times 5400 \mathrm{~K} \mathrm{~m} \mathrm{~s}^{-1}$. The positions of SgrA* (cyan cross), the $20 \mathrm{MC}$, the $50 \mathrm{MC}$, the polar arc (PA) (Bally et al. 1988) (blue rectangle), and the molecular loop (ML, pink ellipse) discussed in paper I, are shown. A scale bar of 15 pc $\left(=0.11^{\circ}\right)$ is displayed in the top right corners. 


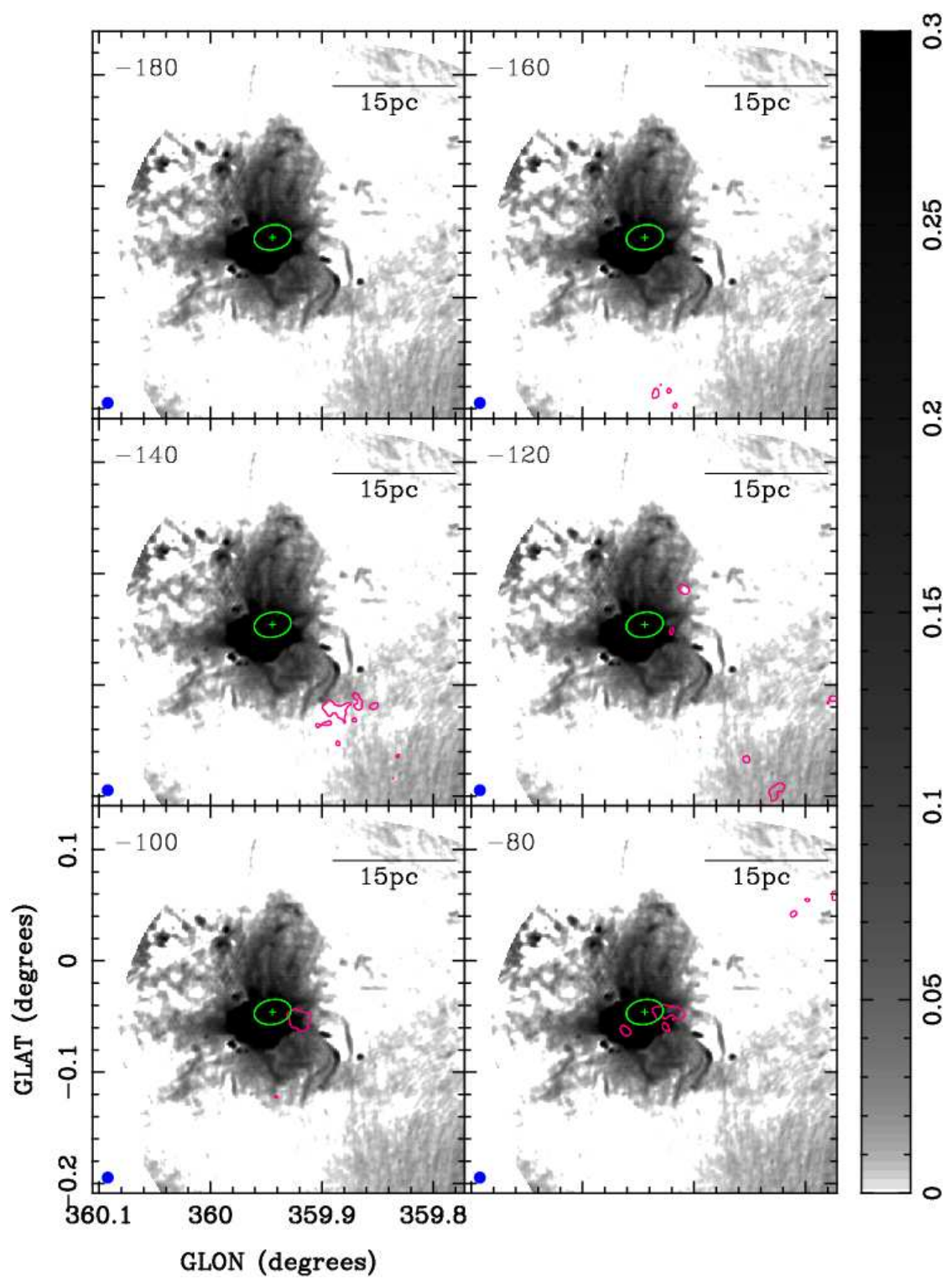

Fig. 2.- $\mathrm{CS}(J=2-1)$ line channel maps (contours) overlaid on archival VLA 20-cm continuum. The $(0,0)$ offset corresponds to $(l, b)=\left(359.939^{\circ},-0.056^{\circ}\right)$. The intrinsic velocity resolution is $2.5 \mathrm{~km} \mathrm{~s}^{-1}$. We show a resolution of $40 \mathrm{~km} \mathrm{~s}^{-1}$ to reduce the number of panels. The beam size of the $\operatorname{CS}(J=2-1)$ line is shown in its original resolution of $21^{\prime \prime}$ (dark blue filled circle in lower left corner). The contour levels are, 5, 10, 20, 30, 40, 50 $\sigma$, where $1 \sigma=0.04 \mathrm{~K}\left(T_{\mathrm{A}} *\right)$. The green ellipse marks the circumnuclear disk (CND). The cross shows the location of SgrA*. The HG-feature discussed in the text is shown with cyan dots. The location of the $20 \mathrm{MC}, 50 \mathrm{MC}$, the HVCC CO0.02-0.20, the PA, and the ML (yellow ellipse) are labeled. 


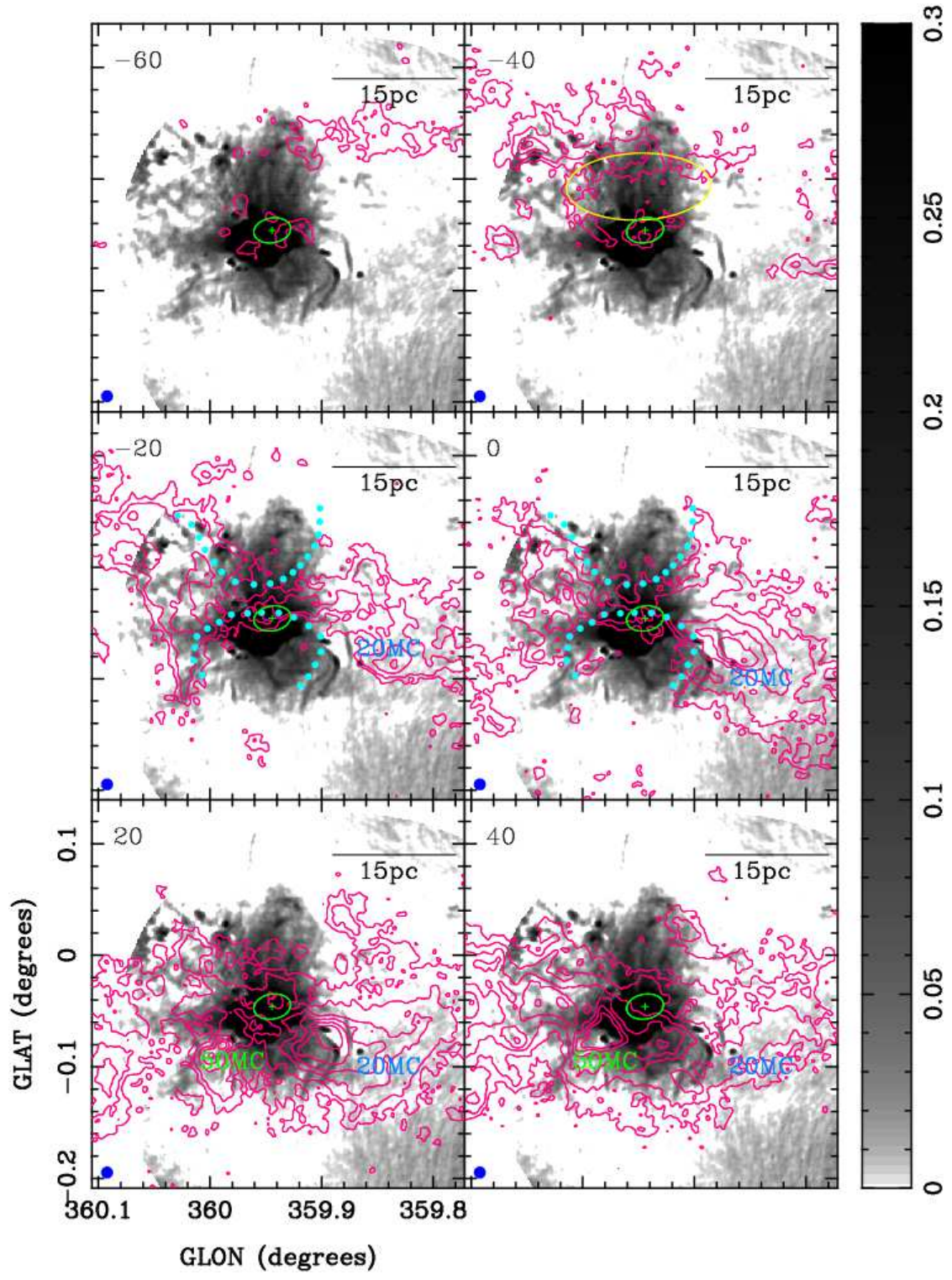

Fig. 2.- Continued. 


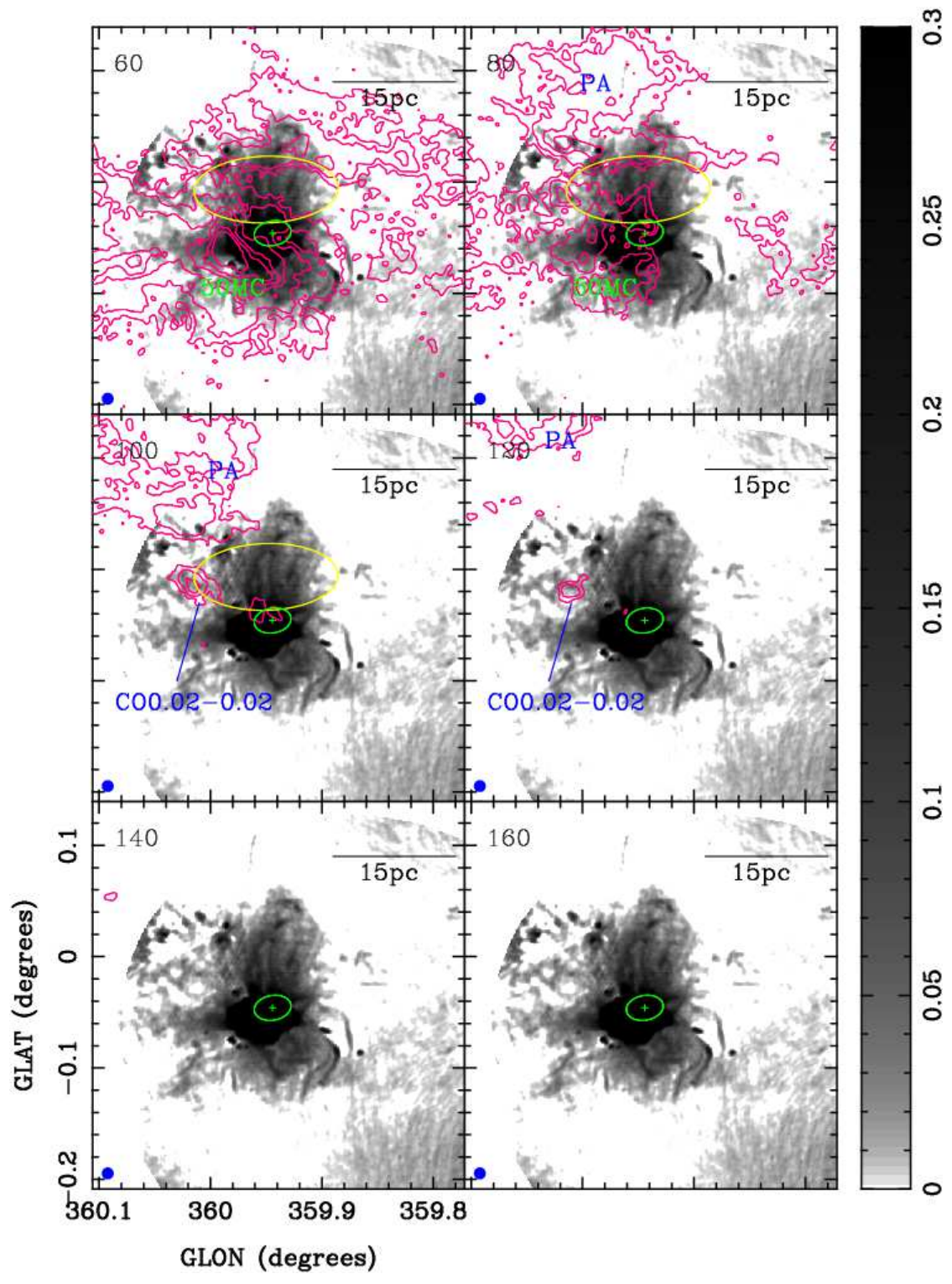

Fig. 2.- Continued. 


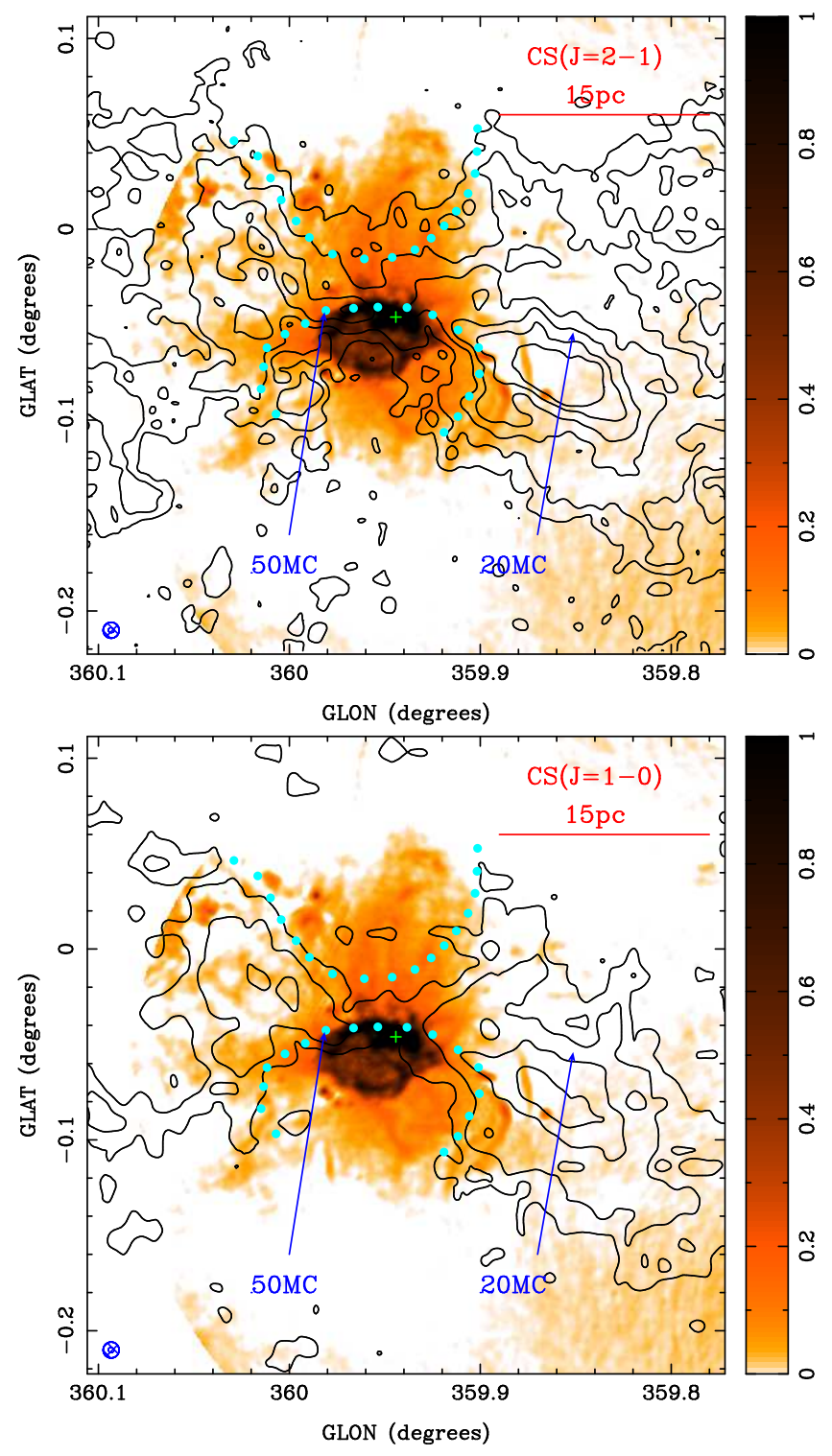

Fig. 3.- Top: $\operatorname{CS}(J=2-1)$ line emission (contours) integrated from $-10 \mathrm{~km} \mathrm{~s}^{-1}$ to $10 \mathrm{~km}$ $\mathrm{s}^{-1}$ overlaid on archival VLA 20-cm continuum (color; Jy/beam). Bottom: CS $(J=1-0)$ line emission (contours) (Tsuboi et al. 1999) integrated from $-10 \mathrm{~km} \mathrm{~s}^{-1}$ to $10 \mathrm{~km} \mathrm{~s}^{-1}$ overlaid on archival VLA 20-cm continuum emission (color). The contour levels are 5, 10, 20, 30, 40, $50 \times 1.47 \mathrm{~K} \mathrm{~km} \mathrm{~s}^{-1}$ for both images. The beam size is $30^{\prime \prime}$ for both images (blue circles in lower left corners). The HG-feature is marked with cyan dots. A scale bar of $15 \mathrm{pc}\left(=0.11^{\circ}\right)$ is shown. 


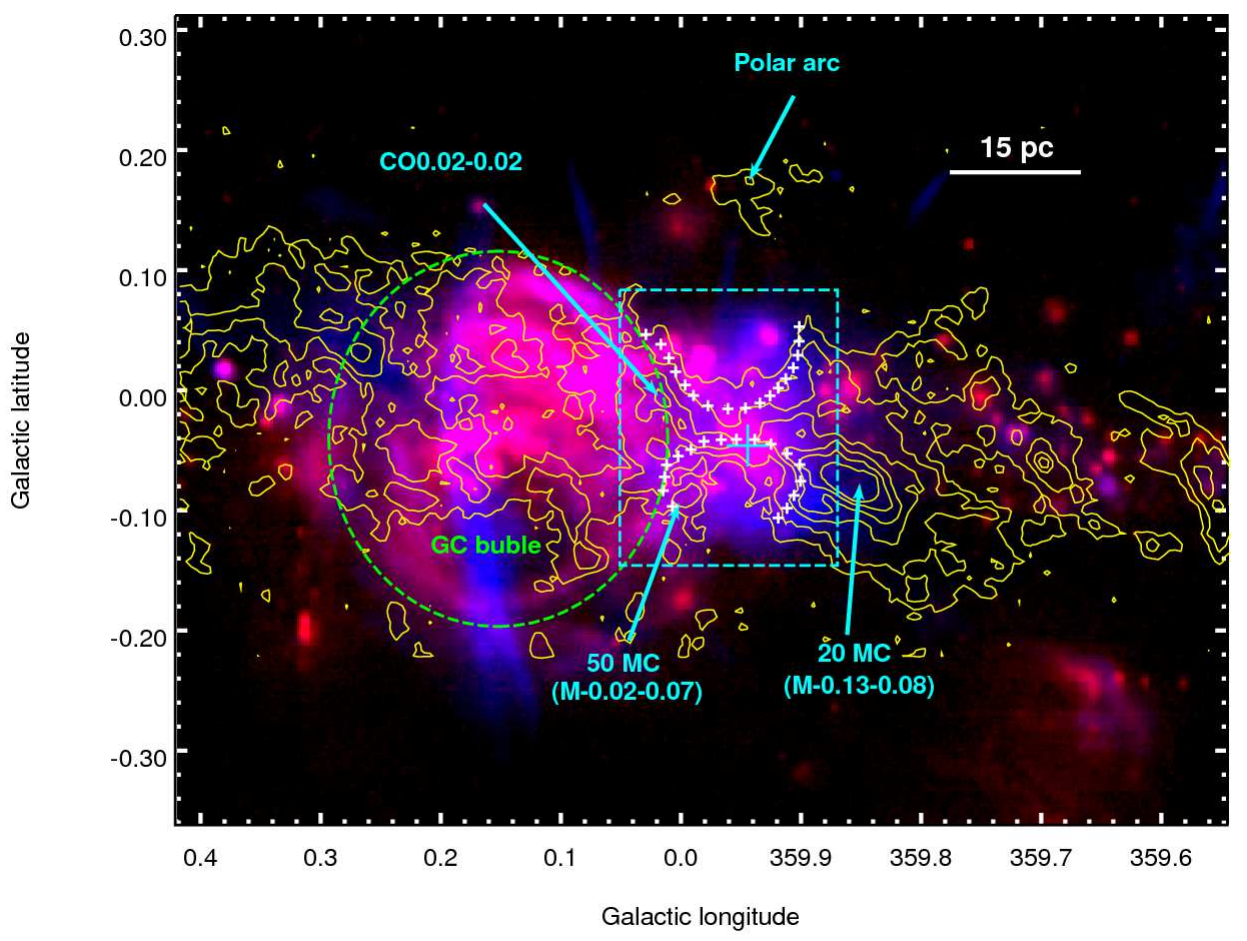

Fig. 4.- Color-composite image of 20-cm VLA map (blue) (Yusef-Zadeh et al. (2004), courtesy from Dr. Yusef-Zadeh) and archival MSX E-band (21 $\mu \mathrm{m})$ map (red) (Price et al. 2001). The $\operatorname{CS}(J=2-1)$ line contour maps integrated over $\pm 10 \mathrm{~km} \mathrm{~s}^{-1}$ are overlaid on it. The hourglass feature is marked with white small crosses and a dashed line box. SgrA* is marked as a large cyan cross. The GC bubble seen in $21 \mu \mathrm{m}$ is marked with a green ellipse. A scale bar of $15 \mathrm{pc}\left(=0.11^{\circ}\right)$ is shown. 


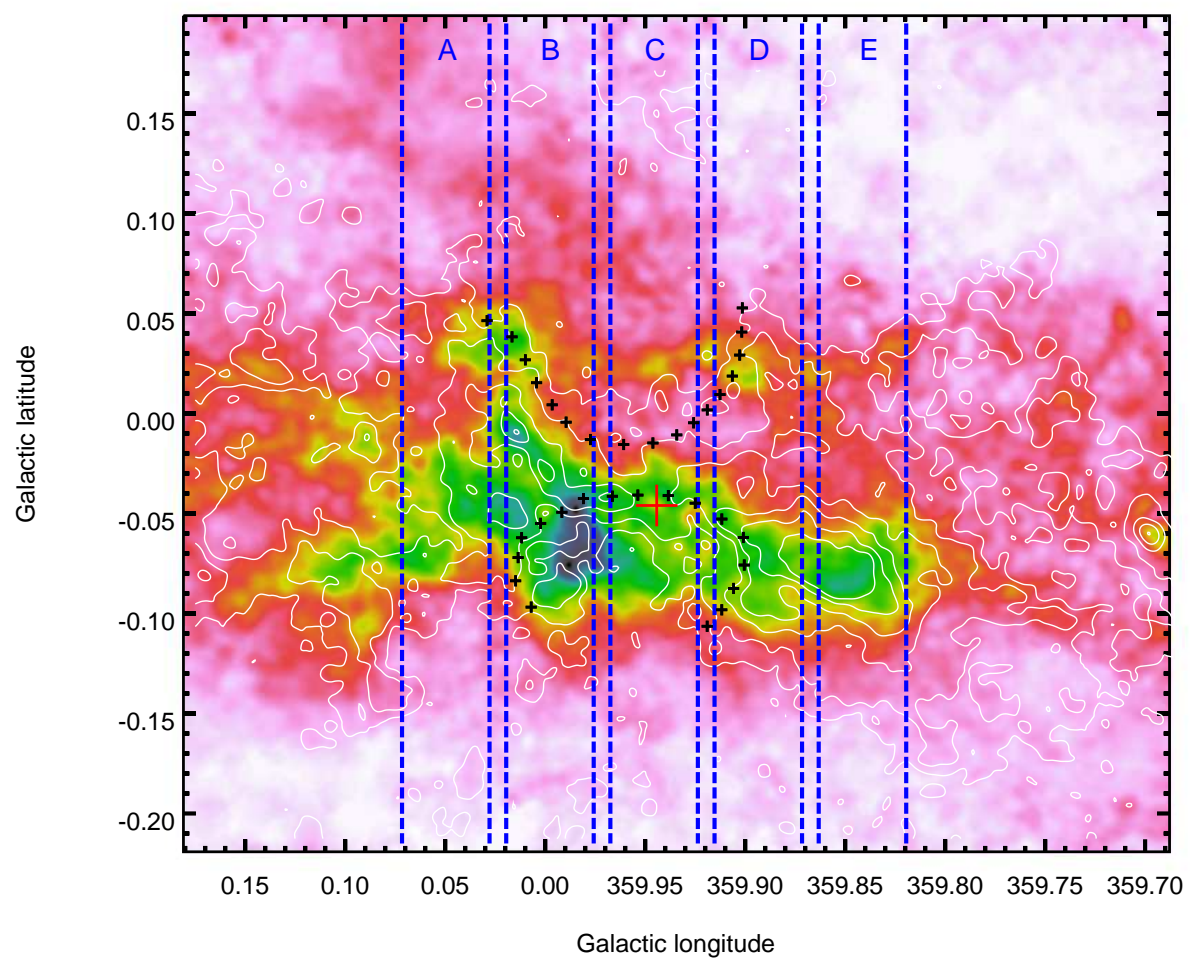

Fig. 5.- Regions made for the latitude velocity diagrams are shown in the CS $(J=2-1)$ line map. Each region covers a width of $2.6^{\prime}$ in longitude. The color map is identical to the one in Figure 1 and the contour image is identical to Figure 3. Regions A, B, C, D, E are marked. The central large red cross marks the position of SgrA*. Small black crosses mark the HG-feature. The spacing between regions corresponds to the convolved beam size of $30^{\prime \prime}$. 

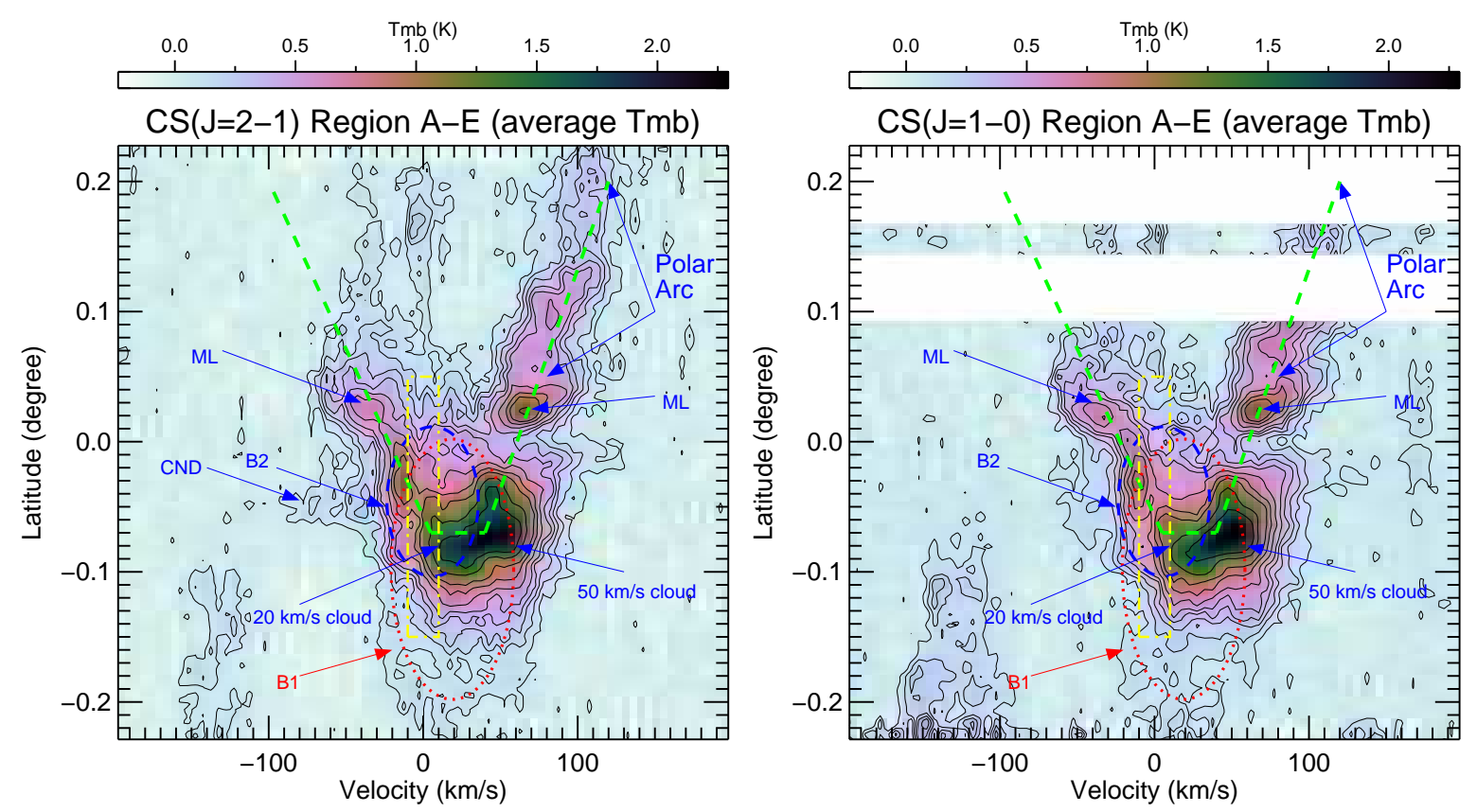

Fig. 6.- Latitude velocity diagrams of region A-E. We use the AIPS task SQASH to collapse longitudinal planes (see Fig. 5) in a cube onto one plane. Diagrams are made by averaging data over the longitude from $l=0.07^{\circ}$ to $-0.18^{\circ}$. The $\mathrm{x}$-axis shows the velocity and the y-axis shows the latitude. The temperature is shown in color and contours in units of brightness temperature $\left(T_{\mathrm{mb}}\right)$. The contours levels are: $\operatorname{CS}(J=2-1): 4,8,12,16,20,27$, $35,42,56,70 \%$ of the peak, where the peak is $2.3 \mathrm{~K}$; $\mathrm{CS}(J=1-0)$ : identical levels but the peak is $1.5 \mathrm{~K}$. The yellow rectangle marks the low-velocity HG-feature. The $50 \mathrm{~km} \mathrm{~s}^{-1}$ and $20 \mathrm{~km} \mathrm{~s}^{-1}$ cloud are located in the Galactic disk. The PA extends out of the Galactic disk to $b \sim 0.23^{\circ}$ with increasing velocity and connects to the Galactic disk via the ML (paper I). The PA is possibly a fossil expanding outflow with a time scale of $6 \times 10^{5}$ years. The B1/B2 labels show the position of an expanding bubble south of the Galactic disk. Other known features of the CND and the HVCC CO0.02-0.02, are labeled as well. 

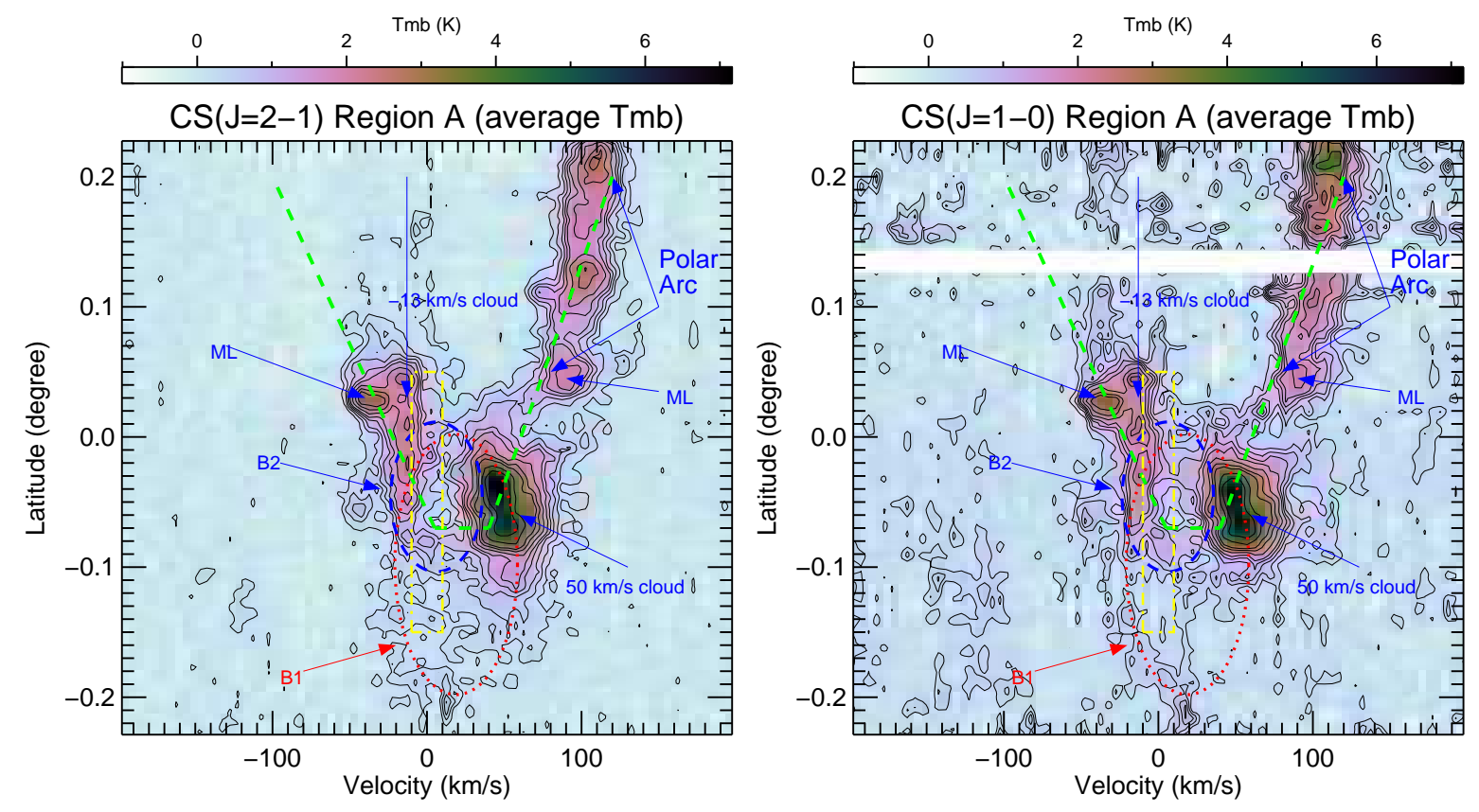

Fig. 7.- Latitude velocity diagrams of region A, which covers the eastern edge of the HGfeature. Identical to Figure 6 but for region A, averaging is performed over the longitude from $l=0.07^{\circ}$ to $0.028^{\circ}$. The contour levels are: $\operatorname{CS}(J=2-1): 4,8,12,16,20,27,35,42$, $56,70 \%$ of the peak, where the peak is $7.2 \mathrm{~K}$; $\mathrm{CS}(J=1-0)$ : identical levels but the peak is 2.2 K. The yellow rectangle marks the eastern side of the HG-feature. The locations of the features are mentioned in the text. 

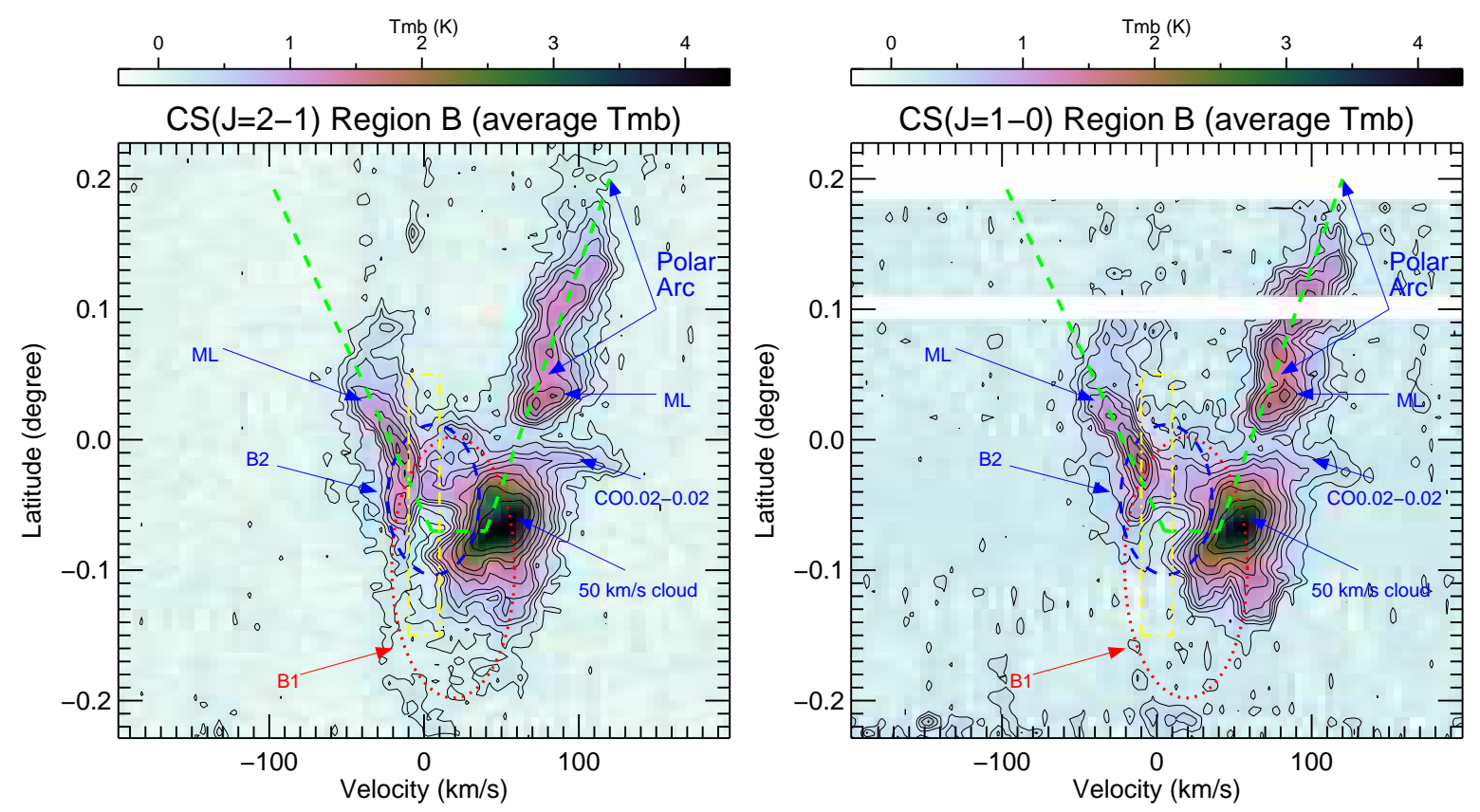

Fig. 8.- Latitude velocity diagrams of region B, which covers the eastern edge of HGfeature. Identical to Figure 6 but for region B, averaging is performed over the longitude from $l=0.019^{\circ}$ to $359.98^{\circ}$. The contour levels are: $\operatorname{CS}(J=2-1): 4,8,12,16,20,27,35$, $42,56,70 \%$ of the peak, where the peak is $4.3 \mathrm{~K}$; $\mathrm{CS}(J=1-0)$ : identical levels but the peak is $3.0 \mathrm{~K}$. The yellow rectangle marks the eastern side of the HG-feature. 

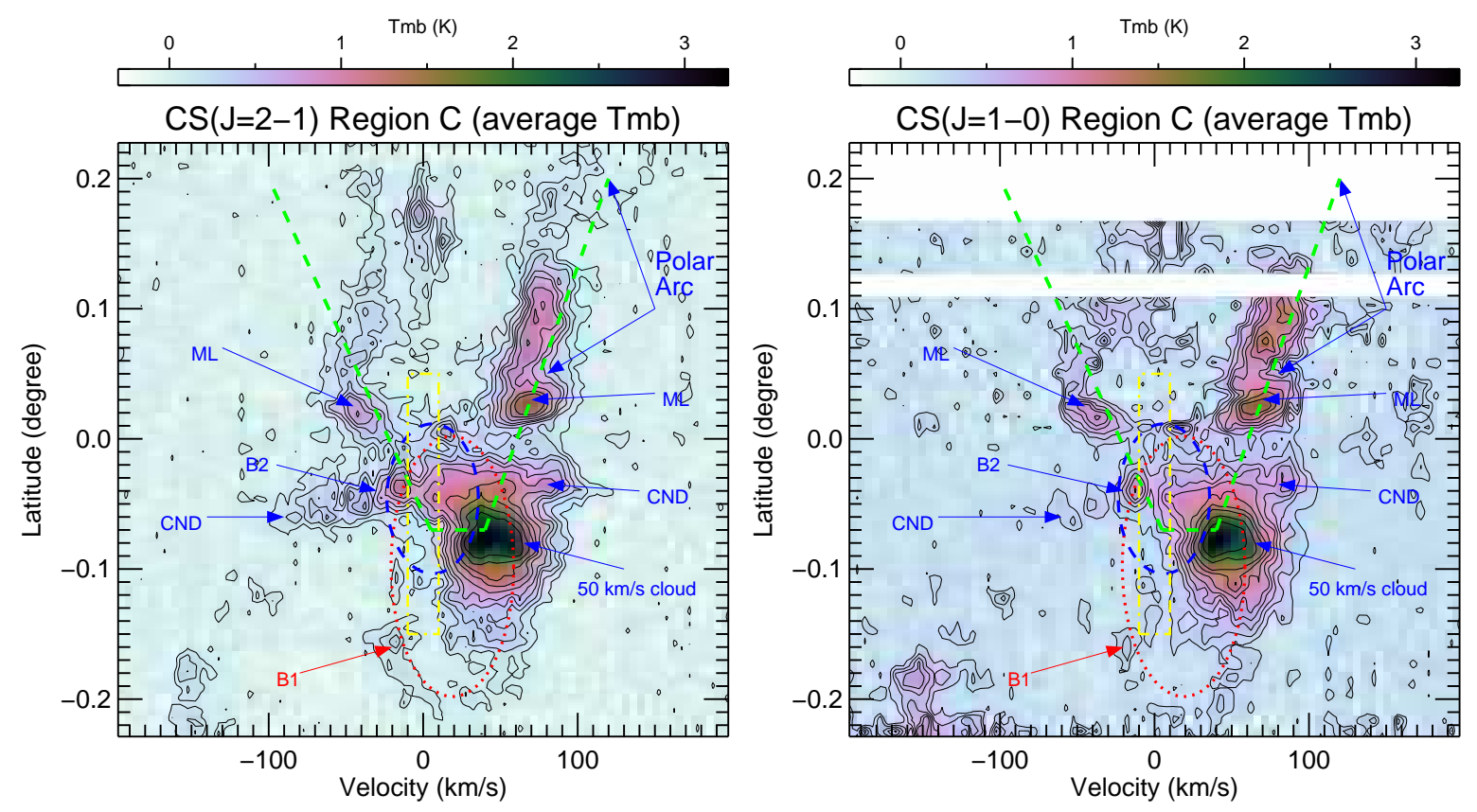

Fig. 9.- Latitude velocity diagrams of region C, which covers SgrA*. Identical to Figure 6 but for region $\mathrm{C}$, averaging is performed over the longitude from $l=359.97^{\circ} 359.92^{\circ}$. The contour levels are: $\operatorname{CS}(J=2-1): 4,8,12,16,20,27,35,42,56,70 \%$ of the peak, where the peak is $3.3 \mathrm{~K}$; $\mathrm{CS}(J=1-0)$ : identical levels but the peak is $2.3 \mathrm{~K}$. The yellow rectangle marks the center of the HG-feature. The features are mentioned in the text. 

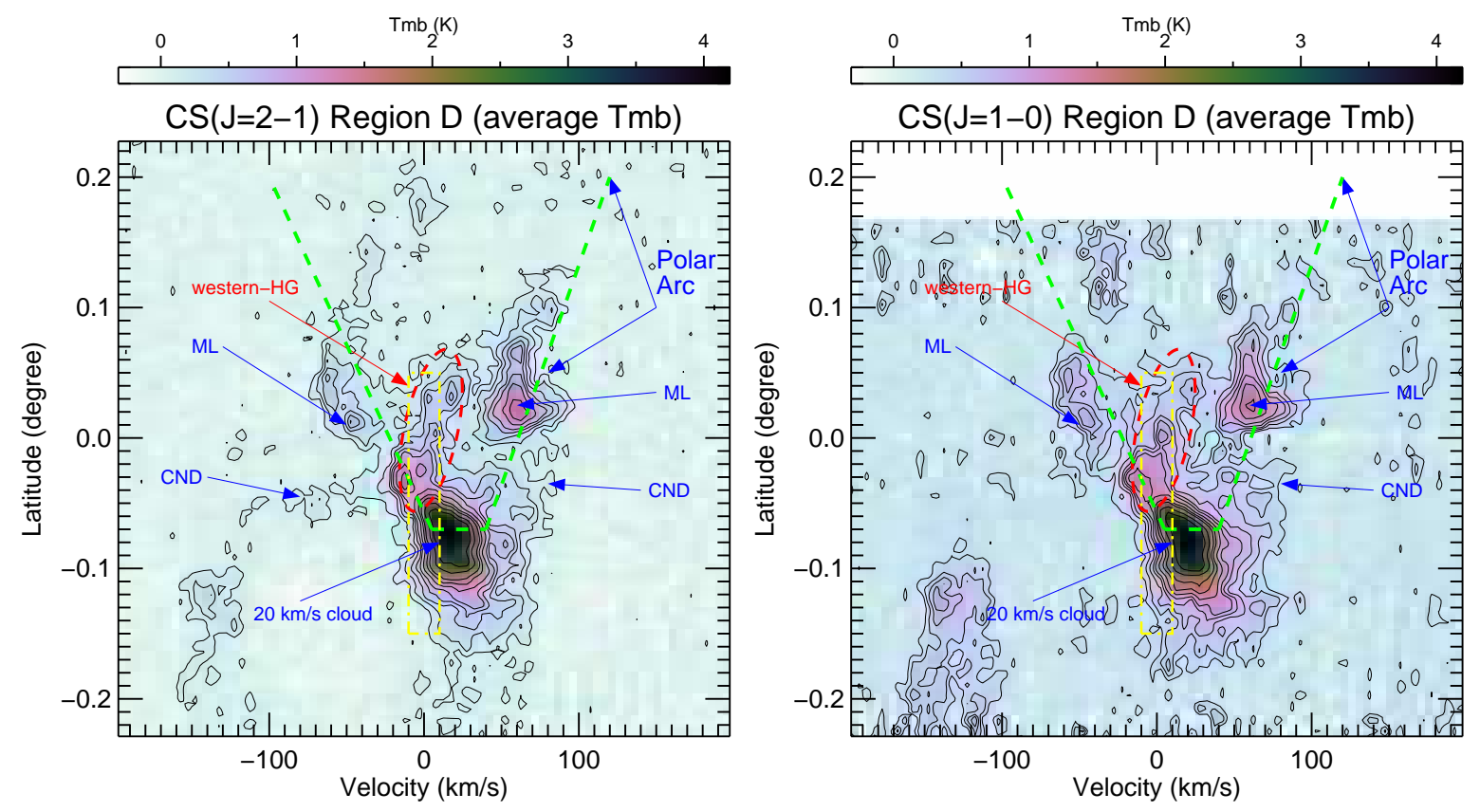

Fig. 10.- Latitude velocity diagrams of region D, which covers the western edge of the HG-feature. Identical to Figure [ but for region D, averaging is performed over the longitude from $l=359.92^{\circ} 359.87^{\circ}$. The contour levels are: $\operatorname{CS}(J=2-1): 4,8,12,16,20,27,35,42$, $56,70 \%$ of the peak, where the peak is $4.2 \mathrm{~K}$; $\mathrm{CS}(J=1-0)$ : identical levels but the peak is $2.6 \mathrm{~K}$. The yellow rectangle marks the western side of the HG-feature. The locations of the features are mentioned in the text. 

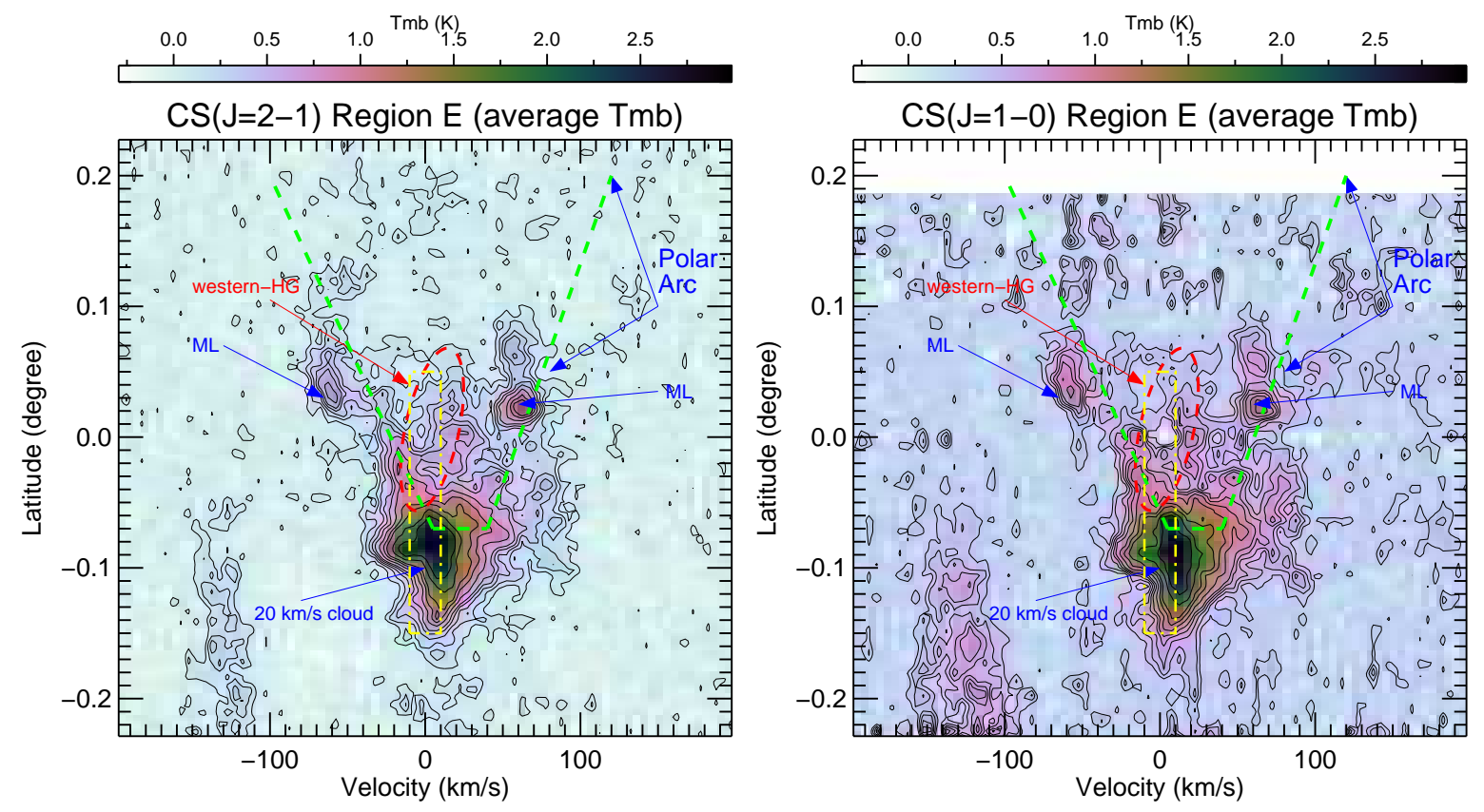

Fig. 11. - Latitude velocity diagrams of region E, which covers the western edge of the HG-feature. Identical to Figure [ but for region E, averaging is performed over the longitude from $l=359.86^{\circ}-0.18^{\circ}$. The contour levels are: $\operatorname{CS}(J=2-1): 4,8,12,16,20,27,35,42$, $56,70 \%$ of the peak, where the peak is $3.0 \mathrm{~K}$; $\mathrm{CS}(J=1-0)$ : identical levels but the peak is $1.8 \mathrm{~K}$. The yellow rectangle marks the western side of the HG-feature. The locations of the features are mentioned in the text. 

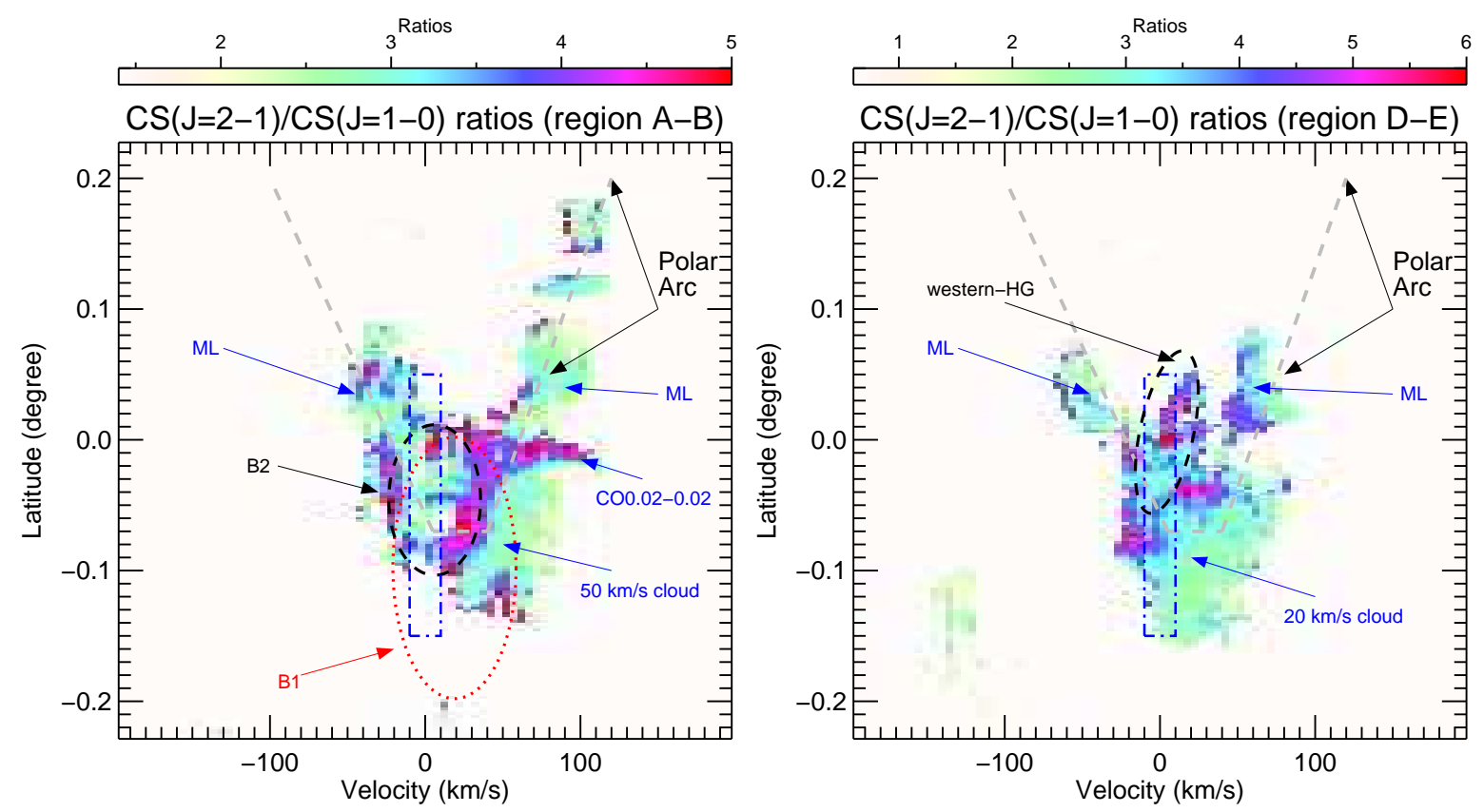

Fig. 12.- Latitude velocity diagrams of the $\operatorname{CS}(J=2-1) / \operatorname{CS}(J=1-0)$ line ratios. Left: ratios are made by averaging the $b-v$ diagrams with region $\mathrm{A}$ and $\mathrm{B}$. Right: ratios are made by averaging the $b-v$ diagrams with region $\mathrm{D}$ and $\mathrm{E}$. The locations of the features are mentioned in the text. The blue rectangle marks the eastern and western side of the HG-feature in the left and right panel, respectively. 


\section{REFERENCES}

Amo-Baladrón, M. A., Martín-Pintado, J., \& Martín, S. 2011, A\&A, 526, A54

Bally, J., Stark, A. A., Wilson, R. W., \& Henkel, C. 1987, ApJS, 65, 13

-. 1988, ApJ, 324, 223

Bland-Hawthorn, J., \& Cohen, M. 2003, ApJ, 582, 246

Bland-Hawthorn, J., Maloney, P. R., Sutherland, R. S., \& Madsen, G. J. 2013, ApJ, 778, 58

Bolatto, A. D., Warren, S. R., Leroy, A. K., et al. 2013, Nature, 499, 450

Burton, W. B., \& Liszt, H. S. 1983, in Astrophysics and Space Science Library, Vol. 105, Surveys of the Southern Galaxy, ed. W. B. Burton \& F. P. Israel, 149-157

Burton, W. B., \& Liszt, H. S. 1992, A\&AS, 95, 9

Carretti, E., Crocker, R. M., Staveley-Smith, L., et al. 2013, Nature, 493, 66

Christopher, M. H., Scoville, N. Z., Stolovy, S. R., \& Yun, M. S. 2005a, ApJ, 622, 346

-. 2005b, ApJ, 622, 346

Coil, A. L., \& Ho, P. T. P. 2000, ApJ, 533, 245

Crocker, R. M., Jones, D. I., Aharonian, F., et al. 2011, MNRAS, 413, 763

Emerson, D. T., \& Graeve, R. 1988, A\&A, 190, 353

Etxaluze, M., Smith, H. A., Tolls, V., Stark, A. A., \& González-Alfonso, E. 2011, AJ, 142, 134

García-Burillo, S., Martín-Pintado, J., Fuente, A., \& Neri, R. 2001, ApJ, 563, L27 
Ghez, A. M., Salim, S., Weinberg, N. N., et al. 2008, ApJ, 689, 1044

Guesten, R., Genzel, R., Wright, M. C. H., et al. 1987, ApJ, 318, 124

Guesten, R., Walmsley, C. M., \& Pauls, T. 1981, A\&A, 103, 197

Guo, F., \& Mathews, W. G. 2012, ApJ, 756, 181

Harris, A. I., Jaffe, D. T., Silber, M., \& Genzel, R. 1985, ApJ, 294, L93

Hartmann, D. H. 1995, ApJ, 447, 646

Henshaw, J. D., Longmore, S. N., Kruijssen, J. M. D., et al. 2016, MNRAS, 457, 2675

Herrnstein, R. M., \& Ho, P. T. P. 2002, ApJ, 579, L83

-. 2005, ApJ, 620, 287

Ho, P. T. P., Jackson, J. M., Barrett, A. H., \& Armstrong, J. T. 1985, ApJ, 288, 575

Hsieh, P.-Y., Ho, P. T. P., \& Hwang, C.-Y. 2015, ApJ, 811, 142

Jackson, J. M., Geis, N., Genzel, R., et al. 1993, ApJ, 402, 173

Koyama, K., Hamaguchi, K., Ueno, S., Kobayashi, N., \& Feigelson, E. D. 1996, PASJ, 48, L87

Lang, C. C., Anantharamaiah, K. R., Kassim, N. E., \& Lazio, T. J. W. 1999, ApJ, 521, L41

Lau, R. M., Herter, T. L., Morris, M. R., Becklin, E. E., \& Adams, J. D. 2013, ApJ, 775, 37

Liu, H. B., Hsieh, P.-Y., Ho, P. T. P., et al. 2012, ApJ, 756, 195

Lutz, D. 1999, in ESA Special Publication, Vol. 427, The Universe as Seen by ISO, ed. P. Cox \& M. Kessler, 623 
Maeda, Y., Baganoff, F. K., Feigelson, E. D., et al. 2002, ApJ, 570, 671

Markoff, S. 2010, Proceedings of the National Academy of Science, 107, 7196

Martín, S., Martín-Pintado, J., Montero-Castaño, M., Ho, P. T. P., \& Blundell, R. 2012, A\&A, 539, A29

Martín, S., Requena-Torres, M. A., Martín-Pintado, J., \& Mauersberger, R. 2008, ApJ, 678,245

Matsushita, S., Muller, S., \& Lim, J. 2007, A\&A, 468, L49

Matsushita, S., Sakamoto, K., Kuo, C.-Y., et al. 2004, ApJ, 616, L55

McGary, R. S., Coil, A. L., \& Ho, P. T. P. 2001, ApJ, 559, 326

McGary, R. S., \& Ho, P. T. P. 2002, ApJ, 577, 757

Mezger, P. G., Zylka, R., Salter, C. J., et al. 1989, A\&A, 209, 337

Mills, E. A. C., Güsten, R., Requena-Torres, M. A., \& Morris, M. R. 2013, ApJ, 779, 47

Montero-Castaño, M., Herrnstein, R. M., \& Ho, P. T. P. 2009, ApJ, 695, 1477

Morris, M., \& Serabyn, E. 1996, ARA\&A, 34, 645

Oka, T., Hasegawa, T., White, G. J., et al. 2008, PASJ, 60, 429

Oka, T., Tanaka, K., Matsumura, S., et al. 2011, in Astronomical Society of the Pacific Conference Series, Vol. 439, The Galactic Center: a Window to the Nuclear Environment of Disk Galaxies, ed. M. R. Morris, Q. D. Wang, \& F. Yuan, 3

Oka, T., White, G. J., Hasegawa, T., et al. 1999, ApJ, 515, 249 
Ozernoy, L. 1996, in IAU Symposium, Vol. 169, Unsolved Problems of the Milky Way, ed. L. Blitz \& P. J. Teuben, 189

Park, S., Muno, M. P., Baganoff, F. K., et al. 2004, ApJ, 603, 548

Pedlar, A., Anantharamaiah, K. R., Ekers, R. D., et al. 1989, ApJ, 342, 769

Ponti, G., Terrier, R., Goldwurm, A., Belanger, G., \& Trap, G. 2010, ApJ, 714, 732

Ponti, G., Morris, M. R., Terrier, R., et al. 2015, MNRAS, 453, 172

Price, S. D., Egan, M. P., Carey, S. J., Mizuno, D. R., \& Kuchar, T. A. 2001, AJ, 121, 2819

Reid, M. J. 1993, ARA\&A, 31, 345

Reid, M. J., Menten, K. M., Brunthaler, A., et al. 2014, ApJ, 783, 130

Requena-Torres, M. A., Güsten, R., Weiß, A., et al. 2012, A\&A, 542, L21

Sawada, T., Ikeda, N., Sunada, K., et al. 2008, PASJ, 60, 445

Scoville, N. Z. 1972, ApJ, 175, L127

Scoville, N. Z., Stolovy, S. R., Rieke, M., Christopher, M., \& Yusef-Zadeh, F. 2003, ApJ, 594,294

Senda, A., Murakami, H., \& Koyama, K. 2003, Astronomische Nachrichten Supplement, 324,151

Serabyn, E., \& Guesten, R. 1987, A\&A, 184, 133

Serabyn, E., Lacy, J. H., \& Achtermann, J. M. 1992, ApJ, 395, 166

Simpson, J. P., Colgan, S. W. J., Cotera, A. S., et al. 2007, ApJ, 670, 1115 
Simpson, J. P., Witteborn, F. C., Cohen, M., \& Price, S. D. 1999, in Astronomical Society of the Pacific Conference Series, Vol. 186, The Central Parsecs of the Galaxy, ed. H. Falcke, A. Cotera, W. J. Duschl, F. Melia, \& M. J. Rieke, 527

Sjouwerman, L. O., Habing, H. J., van Langevelde, H. J., Lindqvist, M., \& Winnberg, A. 1998, in IAU Symposium, Vol. 184, The Central Regions of the Galaxy and Galaxies, ed. Y. Sofue, 129

Sofue, Y. 1995, PASJ, 47, 527

-. 1996, ApJ, 459, L69

-. 2003, PASJ, 55, 445

Su, M., Slatyer, T. R., \& Finkbeiner, D. P. 2010, ApJ, 724, 1044

Sunada, K., Yamaguchi, C., Nakai, N., et al. 2000, in Society of Photo-Optical Instrumentation Engineers (SPIE) Conference Series, Vol. 4015, Society of PhotoOptical Instrumentation Engineers (SPIE) Conference Series, ed. H. R. Butcher, $237-246$

Sunyaev, R. A., Markevitch, M., \& Pavlinsky, M. 1993, ApJ, 407, 606

Tamblyn, P., \& Rieke, G. H. 1993, ApJ, 414, 573

Tsai, A.-L., Matsushita, S., Nakanishi, K., et al. 2009, PASJ, 61, 237

Tsuboi, M., Handa, T., \& Ukita, N. 1999, ApJS, 120, 1

Tsuboi, M., Miyazaki, A., \& Okumura, S. K. 2009, PASJ, 61, 29

Tsuboi, M., Tadaki, K.-I., Miyazaki, A., \& Handa, T. 2011, PASJ, 63, 763 
Veilleux, S. 2004, in Astronomical Society of the Pacific Conference Series, Vol. 320, The Neutral ISM in Starburst Galaxies, ed. S. Aalto, S. Huttemeister, \& A. Pedlar, 277

Veilleux, S., Cecil, G., \& Bland-Hawthorn, J. 2005, ARA\&A, 43, 769

Walter, F., Weiss, A., \& Scoville, N. 2002, ApJ, 580, L21

Wright, M. C. H., Coil, A. L., McGary, R. S., Ho, P. T. P., \& Harris, A. I. 2001a, ApJ, 551, 254

—. 2001b, ApJ, 551, 254

Yokoo, T., Fukue, J., \& Taniguchi, Y. 1993, PASJ, 45, 687

Yusef-Zadeh, F., Hewitt, J. W., \& Cotton, W. 2004, ApJS, 155, 421

Yusef-Zadeh, F., Morris, M., \& Chance, D. 1984, Nature, 310, 557

Zhao, J.-H., Morris, M. R., \& Goss, W. M. 2014, in IAU Symposium, Vol. 303, IAU Symposium, ed. L. O. Sjouwerman, C. C. Lang, \& J. Ott, 364-368

Zubovas, K., King, A. R., \& Nayakshin, S. 2011, MNRAS, 415, L21

This manuscript was prepared with the AAS IATEX macros v5.2. 